\title{
CXCL13 drives spinal astrocyte activation and neuropathic pain via CXCR5
}

\author{
Bao-Chun Jiang, ${ }^{1}$ De-Li Cao, ${ }^{1}$ Xin Zhang, ${ }^{1}$ Zhi-Jun Zhang, ${ }^{1,2}$ Li-Na He, ${ }^{1}$ Chun-Hua Li, ${ }^{1}$ Wen-Wen Zhang, ${ }^{1}$ Xiao-Bo Wu, \\ Temugin Berta, ${ }^{3}$ Ru-Rong Ji, ${ }^{3}$ and Yong-Jing Gao ${ }^{1,4}$
}

1Pain Research Laboratory, Institute of Nautical Medicine, Jiangsu Key Laboratory of Inflammation and Molecular Drug Target, and ²Department of Human Anatomy, School of Medicine, Nantong University, Nantong, China. ${ }^{3}$ Departments of Anesthesiology and Neurobiology, Duke University Medical Center, Durham, North Carolina, USA. ${ }^{4}$ Co-innovation Center of Neuroregeneration, Nantong University, Nantong, China.

\begin{abstract}
Recent studies have implicated chemokines in microglial activation and pathogenesis of neuropathic pain. C-X-C motif chemokine 13 (CXCL13) is a B lymphocyte chemoattractant that activates CXCR5. Using the spinal nerve ligation (SNL) model of neuropathic pain, we found that CXCL13 was persistently upregulated in spinal cord neurons after SNL, resulting in spinal astrocyte activation via CXCR5 in mice. shRNA-mediated inhibition of CXCL13 in the spinal cord persistently attenuated SNLinduced neuropathic pain. Interestingly, CXCL13 expression was suppressed by miR-186-5p, a microRNA that colocalized with CXCL13 and was downregulated after SNL. Spinal overexpression of miR-186-5p decreased CXCL13 expression, alleviating neuropathic pain. Furthermore, SNL induced CXCR5 expression in spinal astrocytes, and neuropathic pain was abrogated in $\mathrm{CXCr}^{-/-}$mice. CXCR5 expression induced by SNL was required for the SNL-induced activation of spinal astrocytes and microglia. Intrathecal injection of CXCL13 was sufficient to induce pain hypersensitivity and astrocyte activation via CXCR5 and ERK. Finally, intrathecal injection of CXCL13-activated astrocytes induced mechanical allodynia in naive mice. Collectively, our findings reveal a neuronal/astrocytic interaction in the spinal cord by which neuronally produced CXCL13 activates astrocytes via CXCR5 to facilitate neuropathic pain. Thus, miR-186-5p and CXCL13/CXCR5-mediated astrocyte signaling may be suitable therapeutic targets for neuropathic pain.
\end{abstract}

\section{Introduction}

Chemokines are a family of small secreted proteins that are wellknown regulators of peripheral immune cell trafficking (1). It has become evident that chemokines are also expressed in the CNS, where they regulate CNS function in both physiological and pathological conditions, including neuronal development, synaptic transmission, and disease-associated neuroinflammation (1-3). Neuropathic pain, caused by lesion or dysfunction of the nervous system, is one of the most intractable human complaints. Activation of spinal glial cells, such as microglia and astrocytes, as part of inflammatory responses in the CNS (neuroinflammation) has been demonstrated as playing a pivotal role in the pathogenesis of neuropathic pain $(4,5)$. Chemokines belong to a very large family with more than 50 members (1). Several chemokines (e.g., CCL2, CCL7, CXCL1, CCL21, and CX3CL1) have been shown to mediate neuronal-glial interactions in the CNS and contribute to both neuropathic pain and inflammatory pain (6-10). It is well established that chemokines such as CCL2, CCL7, CCL21, and CX3CL1

Authorship note: B.C. Jiang, D.L. Cao, X. Zhang, and Z.J. Zhang contributed equally to this work.

Note regarding evaluation of this manuscript: Manuscripts authored by scientists associated with Duke University, The University of North Carolina at Chapel Hill, Duke-NUS, and the Sanford-Burnham Medical Research Institute are handled not by members of the editorial board but rather by the science editors, who consult with selected external editors and reviewers.

Conflict of interest: The authors have declared that no conflict of interest exists. Submitted: March 18, 2015; Accepted: November 19, 2015

Reference information: J Clin Invest. 2016;126(2):745-761. doi:10.1172/JCI81950. are activators of spinal microglia in neuropathic pain conditions, since microglia share features similar to those of macrophages $(6,9,11-13)$. Astrocytes are the most abundant cells in the CNS, have close contacts with synapses, and also produce chemokines (e.g., CCL2 and CXCL1) to maintain neuropathic pain $(7,10)$. However, it is unclear whether chemokines can directly activate astrocytes in neuropathic pain conditions.

$\mathrm{C}-\mathrm{X}-\mathrm{C}$ motif chemokine 13 (CXCL13), also known as B lymphocyte chemoattractant, was originally identified in stromal cells in B cell follicles as regulating homing of B cells and subsets of T cells $(14,15)$. CXCL13 is not expressed in the healthy CNS, but is upregulated in brain and spinal cord under pathological conditions, such as neuroborreliosis, autoimmune demyelination, and primary CNS lymphoma (16-18). In mice with experimental autoimmune encephalomyelitis (EAE), CXCL13 was found in infiltrating dendritic cells in the inflamed brain meninges and spinal cord $(16,17)$. CXCR5 is the receptor for CXCL13 and is expressed on all B cells and a subset of $\mathrm{T}$ cells in blood, lymphatic tissue, and cerebrospinal fluid (CSF) (19, 20). Several studies have shown that CXCL13 levels correlate directly with the number of B cells in brain tissue and CSF (20), while successful treatment of multiple sclerosis (MS) patients is associated with parallel declines of CXCL13 levels and CSF B cell counts (21-23). However, it was also reported that CXCL13deficient animals showed normal CNS B cell recruitment but a mild, self-limited form of EAE $(16,24)$, indicating that CXCL13/ CXCR5 in the CNS may regulate this neuroinflammatory disease via B cell-independent mechanisms. Notably, the role of the CXCL13/CXCR5 axis in neuropathic pain remains unclear. 
In the present study, we used microarray to search for chemokines upregulated in a neuropathic pain condition induced by spinal nerve ligation (SNL) and identified Cxcl13 as the most regulated chemokine gene. We found that SNL is associated with a profound and persistent increase of CXCL13 in neurons of the spinal cord. Interestingly, CXCL13 expression is tightly controlled by a microRNA (miRNA), miR-186-5p, which is downregulated in spinal neurons after SNL. Our results also demonstrate that CXCR5 is persistently increased in activated spinal astrocytes and that SNL-induced astrocyte activation and neuropathic pain are markedly reduced in $\mathrm{CxCr}^{-/-}$mice.

\section{Results}

Cxcl13 is the most upregulated chemokine gene in the spinal cord in a neuropathic pain condition. After SNL, neuropathic pain symptoms such as mechanical allodynia and heat hyperalgesia develop on day 1 and sustain for many weeks (25). To search for novel chemokines that are involved in the maintenance of neuropathic pain, we harvested the ipsilateral spinal cords at 10 days after SNL and performed gene-expression profiling using mouse gene -expression microarrays. The results showed that among the 39,430 Entrez Gene RNAs that could be detected in both SNL and sham-operated mice, 1,139 genes were upregulated by more than 2-fold and 460 genes were downregulated by less than 2-fold relative to the sham group (Supplemental Figure 1A; supplemental material available online with this article; doi:10.1172/JCI81950DS1). Among 37 detectable chemokine genes, 10 ( Cxcl13, Ccl8, Ccl7, Ccl5, Cxcl10, Ccl12, Ccl2, Cxcl1, Ccl3, and Ccl11) were upregulated by more than 3-fold (Supplemental Figure 1, B and C). Strikingly, Cxcl13 was the most dramatically increased gene, with a 47 -fold increase (Supplemental Figure 1C). To validate the microarray result, we conducted quantitative PCR (qPCR) to check the mRNA expression of Cxcl13 and the other 3 chemokines $(\mathrm{Ccl} 7, \mathrm{Ccl} 2$, and $\mathrm{Cxcl1})$ that are involved in neuropathic pain $(6,7,10)$. The fold change of Cxcl13 mRNA (25.1-fold) was much higher than that of other chemokine mRNAs that are known to be upregulated after nerve injury, including $\mathrm{Ccl} 7$ (12.8-fold), Ccl2 (5.1-fold), and Cxcl1 (3.3-fold) (Supplemental Figure 1D). These data suggest that CXCL13 in the spinal cord may play an important role in neuropathic pain.

We then checked the time course of Cxcl13 expression in the ipsilateral dorsal horn after SNL or sham operation. Cxcl13 mRNA was significantly increased at day 1 , peaked at day 10 , and was maintained at day 21 in SNL mice compared with sham-operated mice $(P<0.05$ or 0.001 , Student's $t$ test, Figure $1 \mathrm{~A})$. The mRNA levels did not significantly differ between naive and shamoperated mice at all the time points $(P>0.05,1$-way ANOVA, Figure $1 \mathrm{~A})$. For comparison, we also found that Cxcl13 mRNA was highly expressed in spleen and lymph node and also expressed in brain and dorsal root ganglion (DRG) (Supplemental Figure 2, A and B). ELISA results further confirmed a high expression of CXCL13 protein in the spleen and lymph node (Supplemental Figure 2C) and revealed a significant increase of CXCL13 in the ipsilateral dorsal horn 10 days after SNL $(P<0.001$, Student's $t$ test, Figure $1 \mathrm{~B})$. Interestingly, CXCL13 was also significantly increased in the CSF 10 days after SNL $(P<0.05$, Student's $t$ test, Figure $1 C)$.

CXCL13 is persistently increased in spinal neurons after SNL and contributes to neuropathic pain. To check the expression and distri- bution of CXCL13 in the spinal cord, we did immunofluorescence staining. CXCL13 had low basal expression in the superficial dorsal horn in naive mice (Figure 1D). However, SNL induced a marked increase of CXCL13 immunoreactivity (IR) in the ipsilateral dorsal horn 10 days after SNL (Figure 1E), but not in the contralateral dorsal horn (Figure 1F). CXCL13 IR was abolished after antibody absorption by the immunizing peptide in both spinal cord (Supplemental Figure 3, A and B) and spleen (Supplemental Figure 3, C and D), suggesting the staining might be specific.

To define the cellular localization of CXCL13 in the spinal cord, we performed in situ hybridization using a set of antisense probes (3 probes) for Cxcl13. Cxcl13-positive signal was shown in sections incubated with $\mathrm{Cxcl13}$ antisense probe, but not with scrambled probe (Supplemental Figure 3, E and F). In situ hybridization combined with immunostaining showed that Cxcl13 mRNA was colocalized with the neuronal-specific nuclear protein NeuN (Figure 1, G-I), but not with glial fibrillary acidic protein (GFAP) (Figure 1J) or the ionized calcium-binding adapter molecule (IBA-1) (Figure 1K), suggesting a neuronal expression of Cxcl13 mRNA. In addition, in cultured dorsal horn neurons, CXCL13 was colocalized with microtubule associated protein 2 (MAP2) and CXCL13 IR was observed in both soma and neurites (Figure 1L), further supporting that CXCL13 is expressed by spinal neurons.

We then examined the role of spinal CXCL13 in the pathogenesis of neuropathic pain by testing Cxcl13 shRNA lentivirus vectors for their effects of persistently knocking down CXCL13 expression (LV-Cxcl13 shRNA1-3, Supplemental Table 1). After initial screening, we first chose LV-Cxcl13 shRNA-2 for in vivo study via intraspinal injection, since shRNA-2 showed the best knockdown effects in both Cxcl13 mRNA levels and CXCL13 protein levels in vitro (Supplemental Figure 4, A and B). The shRNA-2 or its negative control lentivirus (LV-NC) was injected 3 days after SNL. Intraspinal injection of LV-Cxcl13 shRNA-2 $\left(2 \times 10^{5} \mathrm{TU}\right)$ did not cause motor function defect or sedation (Supplemental Figure 4, C and D). However, SNL-induced mechanical allodynia was markedly attenuated by this treatment. The antiallodynic effect became evident 7 days after the injection and was maintained for more than 21 days $(P<0.01,2$-way repeated measures ANOVA, Figure 1M). SNL-induced heat hyperalgesia was also alleviated by LV-Cxcl13 shRNA-2 at from 7 days to 21 days $(P<0.001,2$-way repeated measures ANOVA, Figure $1 \mathrm{~N}$ ). Consistent with the behavioral data, qPCR data showed that $\mathrm{Cxcl13}$ in the ipsilateral dorsal horn in LV-Cxcl13 shRNA-2-treated mice was reduced by $63.2 \% \pm 12.7 \%$ compared with the LV-NC-treated mice 21 days after SNL $(P<0.05$, Student's $t$ test $)$.

To further confirm the antinociceptive effect of LV-Cxcl13 shRNA, we also tested another shRNA, LV-Cxcl13 shRNA-3, which showed less knockdown effect compared with LV-Cxcl13 shRNA-2. Nevertheless, LV-Cxcl13 shRNA-3 also attenuated SNL-induced mechanical allodynia and heat hyperalgesia, with a delayed onset (Supplemental Figure 4E) and an early recovery (Supplemental Figure 4F).

We further checked GFP expression and found GFP fluorescence in the ipsilateral dorsal horn 4 days after the injection (Supplemental Figure 5A). Immunostaining showed that GFP was mainly expressed in $\mathrm{NeuN}^{+}$neurons or $\mathrm{GFAP}^{+}$astrocytes and some IBA- $1^{+}$microglia (Supplemental Figure 5, B-D). 
A

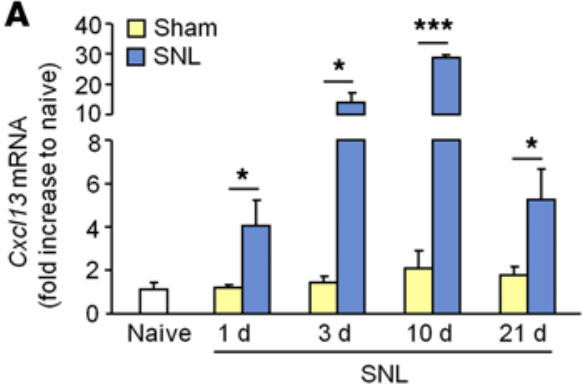

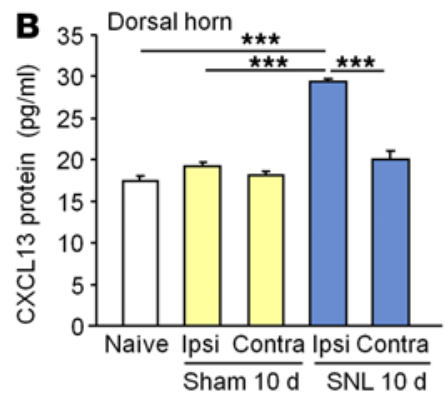
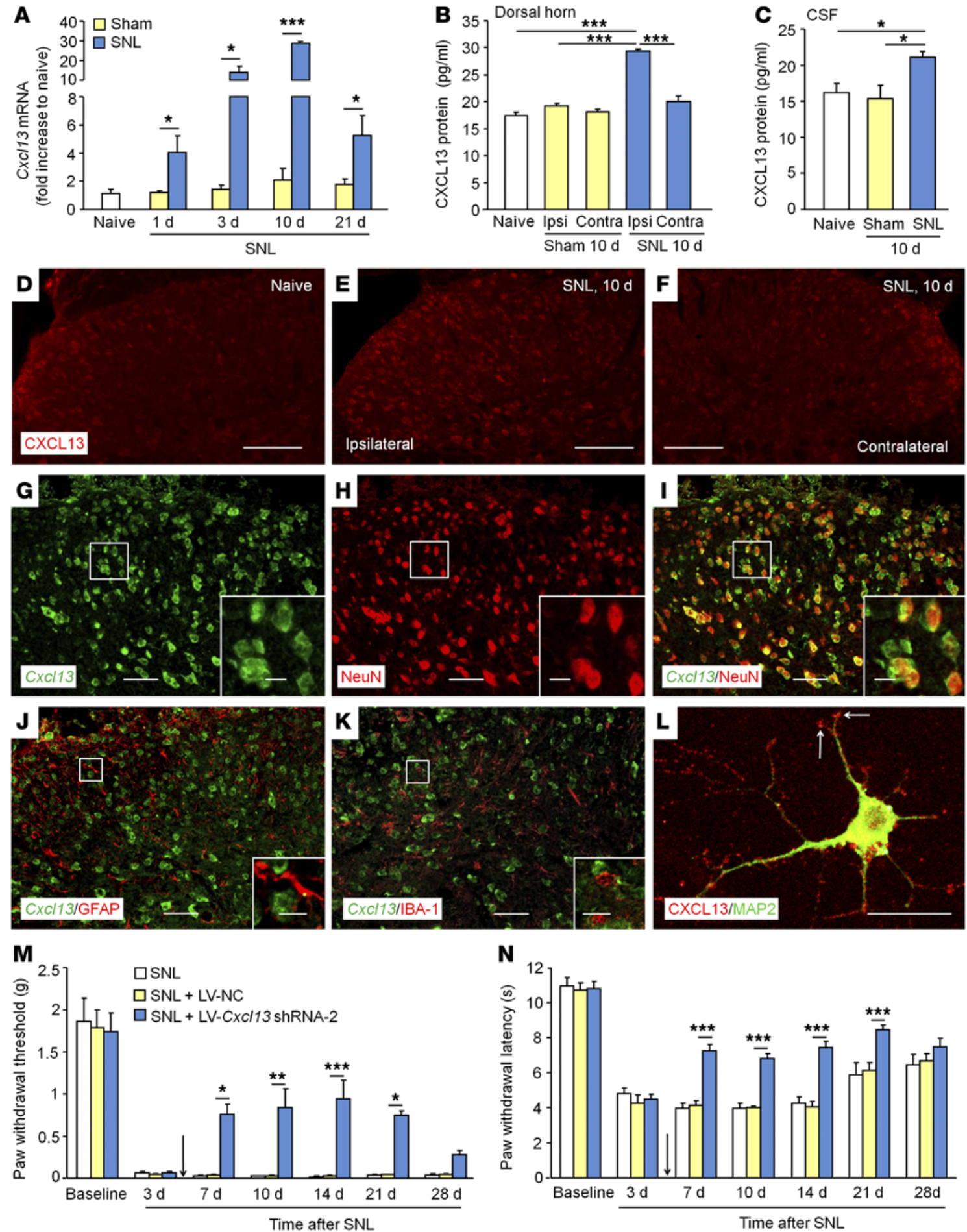

Figure 1. CXCL13 is upregulated in spinal neurons after SNL and contributes to SNL-induced neuropathic pain. (A) The time course of Cxc/13 mRNA expression in the ipsilateral dorsal horn from naive $(n=6)$, sham, and SNL-operated mice. SNL increased Cxc/13 expression at 1 day $(n=3), 3$ days $(n=5), 10$ days $(n=3)$, and 21 days $(n=5)$ compared with sham 1 day $(n=5), 3$ days $(n=3), 10$ days $(n=3)$, and 21 days $(n=5)$, respectively. ${ }^{*} P<0.05$; ${ }^{* * *} P<0.001$, Student's $t$ test. (B) ELISA shows increased CXCL13 protein levels in the ipsilateral dorsal horn after SNL $(n=4)$ compared with sham $(n=5)$ or naive $(n=5)$ animals. ${ }^{* * *} P<0.001$, Student's $t$ test. (C) ELISA shows CXCL13 protein increase in the CSF of mice with neuropathic pain $(n=9)$ compared with sham $(n=7)$ or naive mice $(n=8) .{ }^{*} P<0.05$, Student's $t$ test. $(\mathbf{D}-\mathbf{F})$ Representative images of CXCL13 immunofluorescence in the L5 dorsal horn. CXCL13 IR was low in naive mice (D), but increased in the ipsilateral dorsal horn (E) compared with the contralateral dorsal horn (F) 10 days after SNL. Scale bars: $100 \mu \mathrm{m}$. (G-K) In situ hybridization of $[x c / 13$ mRNA and immunofluorescence staining of NeuN (G-I), GFAP (I), and Iba-1 (K) shows that Cxcl13 mRNA was colocalized with neuronal marker, but not with markers of astrocytes or microglia. Scale bars: $50 \mu \mathrm{m} ; 10 \mu \mathrm{m}$ (insets). (L) Double staining of CXCL13 and neuronal marker MAP2 in cultured dorsal horn neuron. Note the distribution of CXCL13 in neurite terminals (arrows). Scale bar: $25 \mu \mathrm{m}$. (M and $\mathbf{N}$ ) Inhibition of $C x c 113$ by shRNA lentivirus $(n=9)$ attenuated SNL-induced mechanical allodynia (M) and thermal hyperalgesia (N) compared with LV-NC treatment $(n=8)$. Intraspinal injection was performed 3 days after SNL (arrows). ${ }^{*} P<0.05$; ${ }^{* *} P<0.01 ;{ }^{* * *} P<0.001$, 2-way repeated measures ANOVA followed by Bonferroni's test. 
Furthermore, intraspinal injection of LV-NC or LV-Cxcl13 shRNA-2 had no effect on paw withdrawal threshold (PWT) or paw withdrawal latency (PWL) of sham-operated mice (Supplemental Figure 5, E and F). Taken together, these data suggest that CXCL13 in spinal neurons plays a crucial role in the pathogenesis of SNLinduced neuropathic pain.

miR-186-5p targets CXCL13 in spinal dorsal horn neurons. miRNAs are known to inhibit specific gene translation by binding to the 3' UTR sequences of target messenger RNAs (26). To identify potential miRNAs that might target to CXCL13, commonly used computational algorithms (TargetScan, miRDB, and PicTar) were employed. We found that miR-186-5p, miR-1264-3p, and miR-325-3p can potentially target the $3^{\prime}$ UTR of Cxcl13 mRNA (Supplemental Table 2). To check whether these miRNAs are related to the development of neuropathic pain, we examined their expression in the spinal cord after SNL by qPCR. As shown in Figure 2A, the expression of miR-186-5p was gradually declined at post-SNL days 1,3 , and $10(P<0.05$ or 0.01 , 1-way ANOVA), but recovered at days 21 and 28, whereas the expression of miR-1264-3p or miR-325-3p was not significantly changed ( $P>0.05$, 1-way ANOVA). The correlation analysis of the expression of miR-186-5p, miR-1264-3p, and miR-325-3p with that of Cxcl13 showed that only miR-186-5p expression was negatively correlated with Cxcl13 mRNA expression $\left(R^{2}=0.8517, P<0.05\right.$, Figure 2B).

The sequence alignments of miR-186-5p and their binding site in the 3' UTR of Cxcl13 among mammals were highly conserved (Supplemental Figure 6A), especially the seed region, whose conservation score was 100\% (Supplemental Figure 6B), indicating that the regulation of CXCL13 by miR-186-5p may be functionally important for neuropathic pain. Further luciferase reporter assay showed that in Cxcl13 3' UTR vector-transfected HEK293 cells (Supplemental Figure 6C), the renilla/firefly value of luciferase was notably decreased by miR-186-5p mimic, with a $55.2 \%$ decrease by $30 \mathrm{nM}$ miR-186-5p mimic and a $72.9 \%$ decrease by $150 \mathrm{nM}$ miR-186-5p mimic compared with the NC group (Supplemental Figure 6D), indicating the presence of an miR-186-5p target site in Cxcl13 3' UTR. However, the renilla/ firefly value of luciferase was not significantly affected by miR-1264-3p mimic ( $P>0.05$, 1-way ANOVA, Supplemental Figure 6, E and F) or miR-325-3p mimic ( $P>0.05$, 1-way ANOVA, Supplemental Figure 6, G and H).

We then checked the cellular distribution of miR-186-5p in the spinal cord. In situ hybridization analysis showed miR-186-5p-positive cells in spinal cord sections, which were labeled with miR-186-5p miRCURY LNA detection probe, but not with scrambled control probe (Supplemental Figure 7). miR-186-5p was expressed in both dorsal horn and ventral horn of the spinal cord in naive mice (Figure 2C) and was decreased in the ipsilateral dorsal horn 10 days after SNL (Figure 2, D-F). Further double staining showed that miR-186-5p expression was colocalized with NeuN (Figure 2, G-I), but not with GFAP (Figure 2, J-L) or IBA-1 (Figure 2, M-O). Importantly, miR-186-5p was highly colocalized with CXCL13 (Figure 2, P-R). Collectively, these results suggest that miR-186-5p may regulate CXCL13 expression and activity in spinal dorsal horn neurons.

miR-186-5p attenuates pain hypersensitivity by suppressing CXCL13 expression in the spinal cord. To assess the role of miR-186-5p in neuropathic pain, mmu-miR-186 overexpression lentivirus (LVpre-mmu-miR-186) or LV-NC was intraspinally injected into the L5 dorsal horn 3 days after SNL. qPCR showed that miR-186-5p was increased by $41 \% \pm 9 \%(P<0.01$, vs. LV-NC, Student's $t$ test $)$ 7 days after injection. Overexpression of miR-186-5p by LV-premmu-miR-186 alleviated mechanical allodynia and heat hyperalgesia at days 7,10 , and $14(P<0.001,2$-way repeated measures ANOVA, Figure 3, A and B). Intraspinal injection of LV-pre-mmumiR-186 in normal animals did not affect PWT or PWL (Supplemental Figure 8). To further confirm the effect of miR-186-5p in neuropathic pain, we injected vehicle intrathecally (i.t.), scrambled mimic (NC, $5 \mu \mathrm{g}$ ), or miR-186-5p mimic ( $5 \mu \mathrm{g}$ ) daily for 3 days in mice with SNL (from day 8 to day 10 after SNL). Mechanical allodynia and heat hyperalgesia were tested 6 hours after each injection. miR-186-5p mimic alleviated mechanical allodynia $(P<0.001$, Figure 3C) and heat hyperalgesia $(P<0.001$, Figure 3D). We harvested the spinal cord after the behavioral testing on day 10 and found that CXCL13 expression was suppressed by $48.6 \%$ after the injection of miR-186-5p mimic compared with vehicle injection $(P<0.05$, vs. vehicle, Student's $t$ test, Figure $3 \mathrm{E})$. In addition, the inhibitory effect of miR-186-5p mimic could be rescued by i.t. injection of CXCL13 (Supplemental Figure 9). Thus, miR-186-5p upregulation attenuated neuropathic pain at least partly by regulating CXCL13 protein expression, although miR-186-5p is also known to regulate the expression of other genes (27).

Next, we examined whether inhibition of miR-186-5p could induce CXCL13-dependent pain hypersensitivity. We i.t. injected miR-186-5p inhibitor into naive mice daily for 3 days and checked pain behavior 6 hours after each injection. The i.t. administration of miR-186-5p inhibitor ( 1 or $5 \mu \mathrm{g}$ ) significantly induced mechanical hyperalgesia and heat hyperalgesia $(P<0.001,2$-way repeated measures ANOVA, Figure 3, F and G) in naive mice. Furthermore, i.t. injection of miR-186-5p inhibitor $(5 \mu \mathrm{g})$ also increased CXCL13 levels $(34.4 \pm 5.4 \mathrm{pg} / \mathrm{ml}$ in miR-186-5p inhibitor-treated group vs. $25.2 \pm 1.8 \mathrm{pg} / \mathrm{ml}$ in NC group, $P<0.05$, Student's $t$ test). Importantly, i.t. injection of CXCL13 neutralizing antibody effectively attenuated miR-186-5p inhibitor-induced mechanical allodynia $(P<0.05$, 2-way repeated measures ANOVA, Figure 3H). Moreover, i.t. injection of NC, miR-186-5p mimic, or inhibitor did not induce motor dysfunction or sedative behavior (Supplemental Figure 10). Collectively, these results suggest that miR-186-5p is a tonic endogenous inhibitor of pain and that loss of this inhibitor is sufficient to increase CXCL13 expression and induce pain hypersensitivity.

miR-186-5p downregulation and CXCL13 upregulation after $S N L$ are mediated by NMDA receptor in the spinal cord. We then asked what is responsible for the miR-186-5p downregulation and CXCL13 upregulation in the spinal cord after SNL. Since spinal NMDA receptor (NMDAR) plays a pivotal role in nerve injury-induced central sensitization and neuropathic pain (28), we tested the involvement of NMDAR in these regulations. The i.t. pretreatment of MK-801 (10 nmol), a noncompetitive NMDA glutamate receptor antagonist $(29,30)$, prevented both the downregulation of miR-186-5p (Figure 3I) and the upregulation of CXCL13 following SNL (Figure 3J). In contrast, i.t. injection of NMDA ( $1 \mathrm{nmol}$ ) decreased miR-186-5p expression (Figure 3K) and increased CXCL13 expression (Figure 3L) in the spinal cord of naive mice. These data suggest that SNL-induced 
A

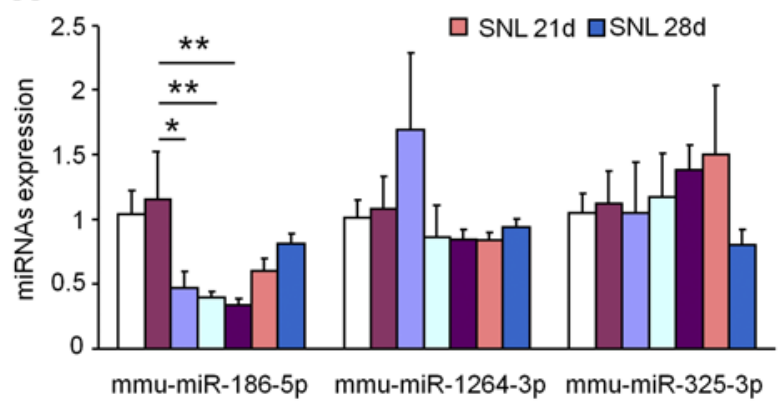

B $\rightarrow$ miR-186-5p $=m i R-1264-3 p \Delta$ miR-325-3p

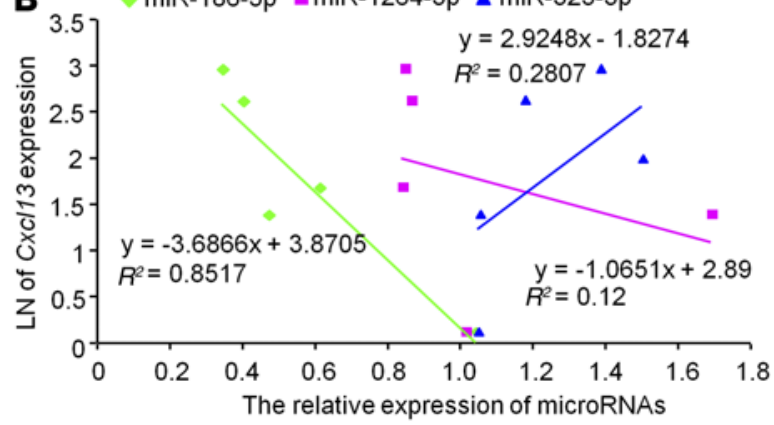

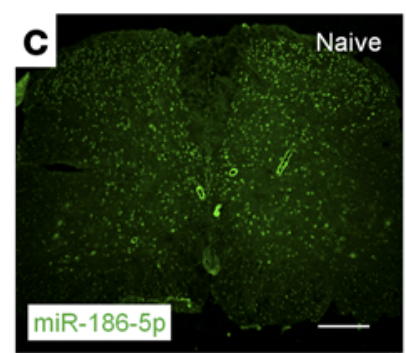
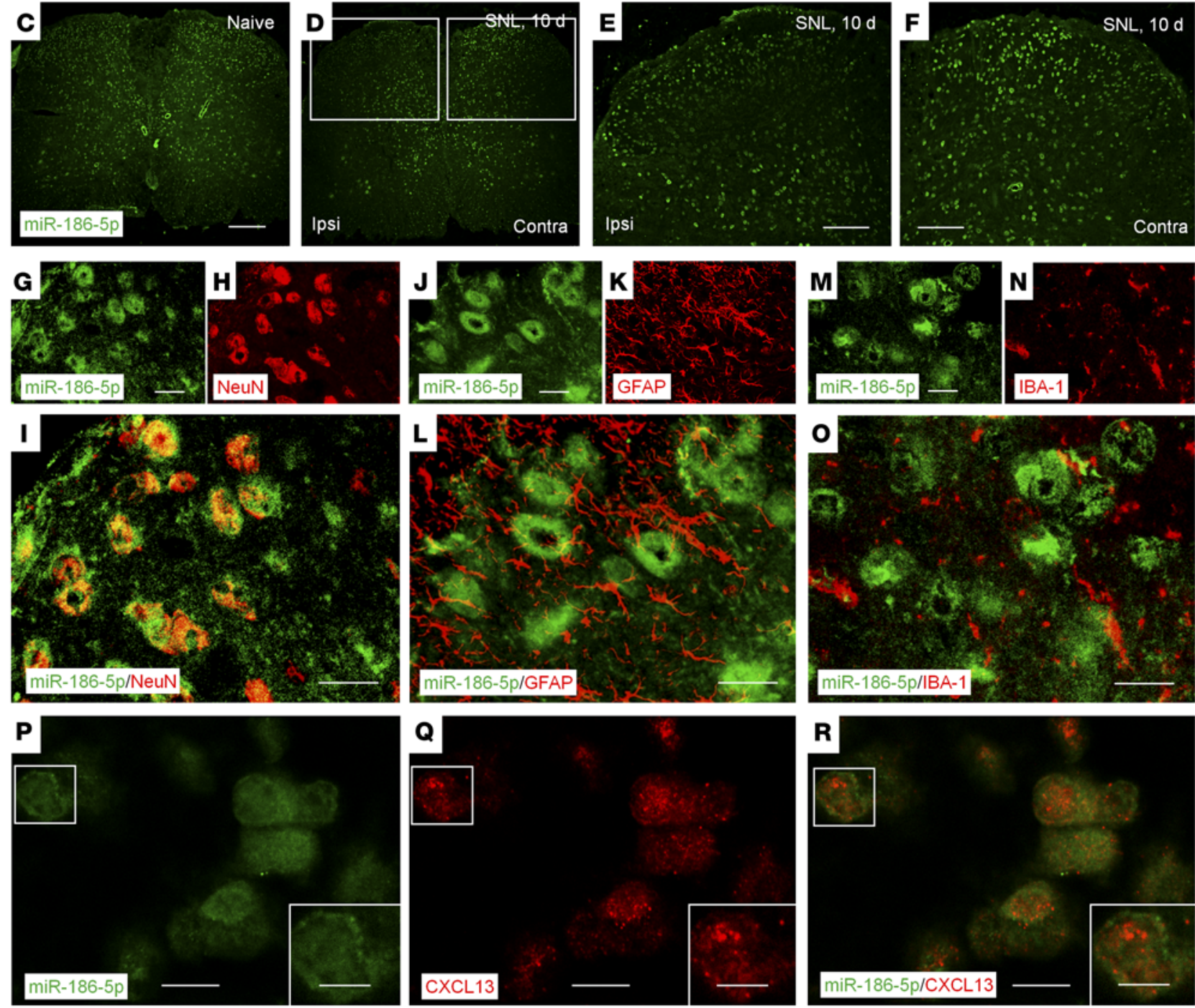

Figure 2. miR-186-5p is downregulated in the spinal cord after SNL and colocalized with CXCL13 in spinal neurons. (A) The expression of miR186-5p was decreased at days 1,3 , and 10 after SNL, while the expression of miR-1264-3p or miR-325-3p was not significantly changed after SNL. ${ }^{*} P<0.05 ;{ }^{*} P<0.01,1$-way ANOVA followed by Bonferroni's test. $n=3-6$ mice/group. (B) Correlation analysis of the expression of Cxc/13 mRNA with the expression of miR-186-5p, miR-1264-3p, or miR-325-3p, respectively. The natural logarithm (LN) Cxc/13 mRNA expression in the spinal cord was negatively correlated with the expression of miR-186-5p. $R^{2}=0.8517 ; P<0.05$. Diamonds, squares, and triangles represent groups of individuals (mean value) across time. (C and D) FISH shows the expression of miR-186-5p in naive (C) and SNL (D) mice. Scale bar: $200 \mu \mathrm{m}$. (E and F) High magnification image of $\mathbf{D}$, indicated in the white boxes of $\mathbf{D}$. Scale bars: $100 \mu \mathrm{m}$. miR-186-5p was decreased in the ipsilateral dorsal horn (E, ipsi) compared with the contralateral dorsal horn (F, contra) 10 days after SNL. (G-0) FISH for miR-186-5p combined with immunofluorescence staining for cell markers NeuN (G-I), GFAP (J-L), and Iba-1 (M-0), respectively. Scale bars: $20 \mu \mathrm{m}$. miRNA-186-5p expression was only detected in NeuNlabeled neurons (I) in spinal dorsal horn. (P-R) Combined FISH and immunofluorescence show that miR-186-5p (P) was colocalized with CXCL13 (Q and R). Scale bars: $10 \mu \mathrm{m} ; 5 \mu \mathrm{m}$ (insets). 

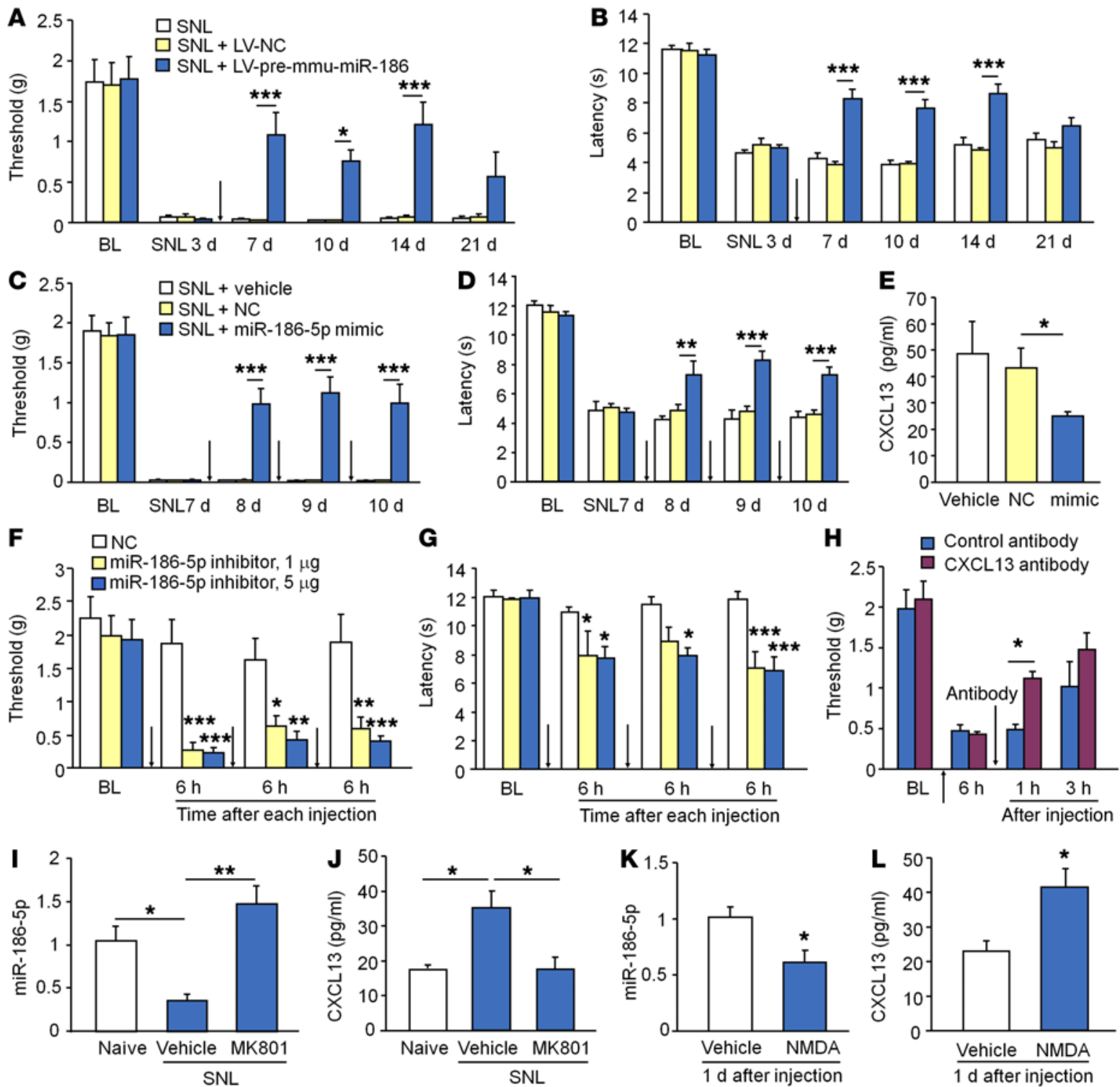

Figure 3. miR-186-5p inhibits neuropathic pain via suppressing CXCL13 expression in the spinal cord. (A and B) Intraspinal infusion of mmupre-miR-186 expressing lentivirus 3 days after SNL alleviated SNL-induced mechanical allodynia (A) and heat hyperalgesia (B) at days 7, 10, and 14. ${ }^{*} P<0.05$; ${ }^{* *} P<0.001$, 2-way repeated measures ANOVA followed by Bonferroni's test. $n=7$ for LV-NC; $n=8$ for SNL or LV-pre-mmu-miR-186 group. BL, baseline. (C and $\mathbf{D}$ ) Injection (i.t.) of miR-186-5p mimic daily for 3 days (from day 8 to day 10 after SNL) attenuated SNL-induced mechanical allodynia (C) and heat hyperalgesia (D). ${ }^{*} P<0.01$, ${ }^{* * *} P<0.001,2$-way repeated measures ANOVA followed by Bonferroni's test. $n=9$ for NC and $n=10$ for miR-186 mimic. (E) ELISA results show decreased CXCL13 levels in the spinal cord of miR-186-5p mimic-treated animals. ${ }^{*} P<0.05$, Student's $t$ test. $n=6$ mice/group. (F and $\mathbf{G}$ ) Injection (i.t.) of miR-186-5p inhibitor induced mechanical allodynia (F) and heat hyperalgesia (G) in naive mice. ${ }^{*} P<0.05$; ${ }^{* *} P<0.01$; ${ }^{* *} P<0.001$, miR-186-5p inhibitor vs. NC, 2-way repeated measures ANOVA followed by Bonferroni's test. $n=5$ mice/group. (H) CXCL13 neutralizing antibody attenuated miR-186-5p inhibitor-induced mechanical allodynia. ${ }^{*} P<0.05,2$-way repeated measures ANOVA followed by Bonferroni's test. $n=5$ mice/group. (I) Injection (i.t.) of MK801 before SNL prevented SNL-induced downregulation of miR-186-5p in the spinal cord. ${ }^{*} P<0.05$; ${ }^{* *} P<0.01$, 1-way ANOVA followed by Bonferroni's test. $n=5$ mice/group. (J) Injection (i.t.) MK801 also inhibited SNL-induced upregulation of CXCL13. ${ }^{*} P<0.05$, 1-way ANOVA followed by Bonferroni's test. $n=4-6$ mice/group. (K) Injection (i.t.) of NMDA in naive mice decreased miR-186-5p expression. ${ }^{*} P<0.05$, Student's $t$ test. $n=4-5$ mice/group. (L) Injection (i.t.) NMDA also increased CXCL13 expression. ${ }^{*} P<0.05$, Student's $t$ test. $n=6$ mice/group.

miR-186-5p downregulation and CXCL13 upregulation are mediated, at least partly, by NMDAR.

SNL increases CXCR5 $\mathrm{MRNA}$ and protein expression in spinal astrocytes. It has been reported that CXCR5 was the sole receptor for CXCL13 and vice versa (20). Cxcr5 mRNA was expressed in the brain, spinal cord, and DRG and was highly expressed in spleen and lymph node (Supplemental Figure 11, A and B). Western blot also confirmed the expression of CXCR5 in the spinal cord, spleen, and lymph node (Supplemental Figure 11, C and D). SNL induced rapid and persistent Cxcr5 mRNA upregulation, which started at day 1 and was maintained at day $21(P<0.05$ or 0.001 , SNL vs. sham, Student's $t$ test, Figure 4A). Western blot showed 

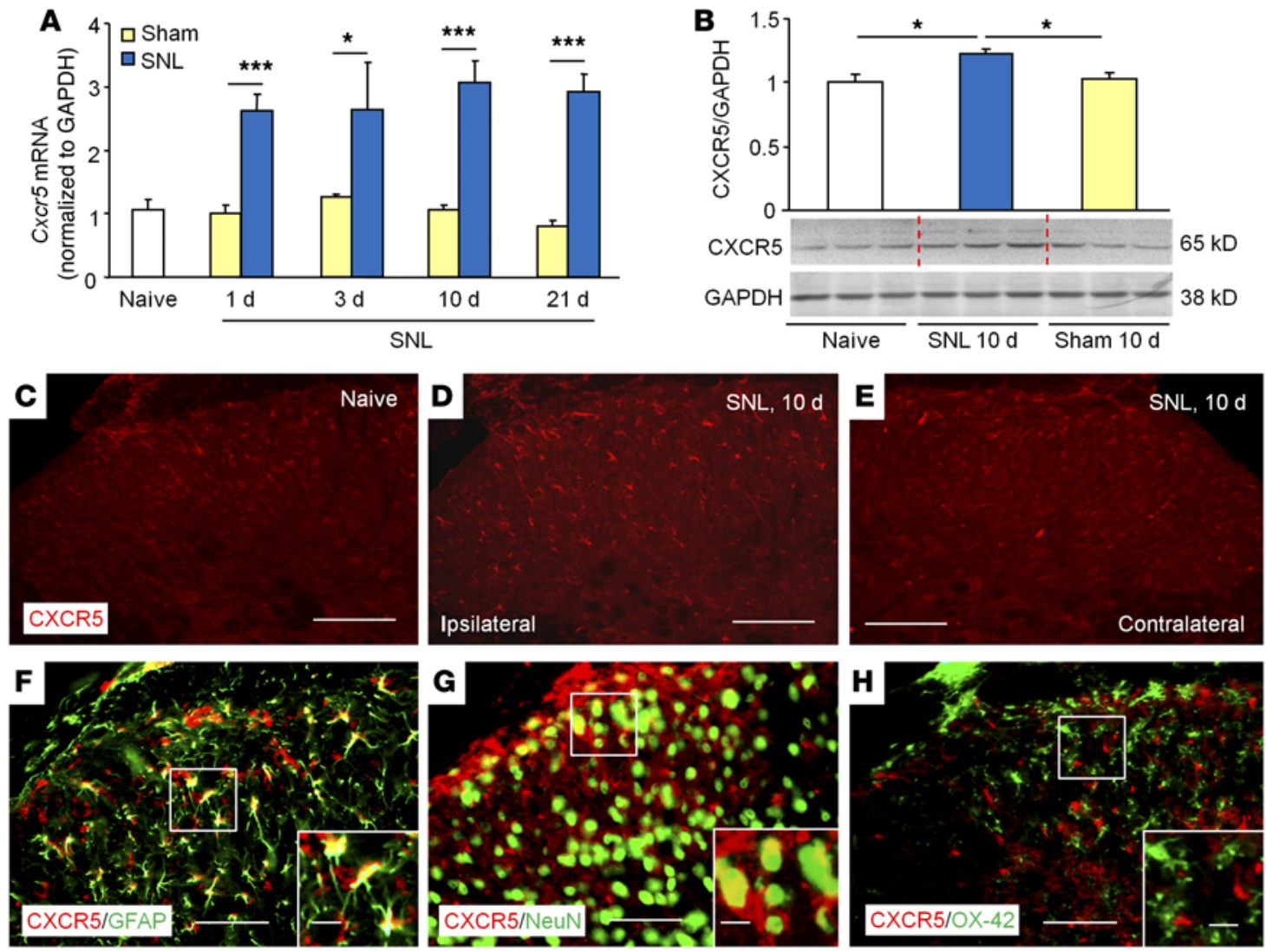

Figure 4. CXCR5 expression is increased in spinal astrocytes after SNL. (A) Time course of $C x c r 5$ mRNA expression in the ipsilateral dorsal horn in naive $(n=6)$, sham-operated $(n=5)$, and SNL mice. SNL increased Cxcr5 expression at 1 day $(n=5), 3$ days $(n=3), 10$ days $(n=4)$, and 21 days $(n=4)$ compared with sham. ${ }^{*} P<0.05$; ${ }^{* *} P<0.001$, Student's $t$ test. (B) Western blotting shows the increase of CXCR5 protein in the spinal cord 10 days after SNL. ${ }^{*} P<0.05$, Student's $t$ test. $n=3$ mice/group. (C-E) Representative images of CXCR5 immunofluorescence in the spinal cord from naive and SNL mice. CXCR5 IR was low in naive mice (C), increased in the ipsilateral dorsal horn of SNL mice (D), and unchanged in the contralateral dorsal horn of SNL mice (E). Scale bars: $100 \mu \mathrm{m}$. (F-H) Double staining shows that CXCR5 is mainly colocalized with GFAP (F), rarely with NeuN (C), but not with OX-42 (H) in the dorsal horn of spinal cord 10 days after SNL. Scale bars: $50 \mu \mathrm{m} ; 10 \mu \mathrm{m}$ (insets).

that CXCR5 protein level was significantly increased in SNL mice at 10 days $(P<0.05$, SNL vs. naive or sham, Student's $t$ test, Figure $4 \mathrm{~B})$. Immunostaining further revealed that CXCR5 had low expression in the dorsal horn in naive mice (Figure 4C). CXCR5 IR was markedly increased in ipsilateral dorsal horn (Figure 4D), but not in contralateral dorsal horn (Figure 4E) 10 days after SNL. Double-immunofluorescence staining showed that CXCR5 was mainly colocalized with the GFAP $(96.6 \%$, Figure $4 \mathrm{~F})$ and partially with $\operatorname{NeuN}(2.3 \%$, only in lamina I, Figure $4 \mathrm{G})$, but not with OX-42 (Figure $4 \mathrm{H}$ ) in the dorsal horn of spinal cord.

Deletion or inhibition of CXCR5 persistently attenuates $S N L$ induced pain hypersensitivity. To determine the role of CXCR5 in pain sensation, we first checked acute pain behaviors and motor function in WT mice and Cxcr5 KO mice. KO mice (Supplemental Figure 12A) showed normal gross anatomy (Supplemental Figure 12B). Spinal cord CXCR5 expression was lost in KO mice, as shown by immunostaining (Supplemental Figure 12, C and D). KO mice also showed normal distribution patterns in the spinal cord dorsal horn of the neurochemical markers NeuN (Supplemental Figure 12, E and F) and PKC $\gamma$ (Supplemental Figure 12, $\mathrm{G}$ and $\mathrm{H}$ ) and normal innervations of the primary afferents, labeled with CGRP and IB4 (Supplemental Figure 12, I-L). Acute thermal sensitivity, as assessed by hot water immersion (Figure $5 \mathrm{~A}$ ) and radiant heat (Hargreaves, Figure 5B), was indistinguishable in WT and Cxcr5 KO mice ( $P>0.05$, Student's $t$ test). Acute mechanical sensitivity, as evaluated by von Frey filament (Figure $5 \mathrm{C}$ ), was also comparable between the 2 genotypes $(P>0.05$, Student's $t$ test). The rotarod test revealed similar falling latency in WT and Cxcr5 KO mice ( $P>0.05$, Student's $t$ test, Figure 5D). These data indicate that $C x c r 5$ deletion did not cause deficits in acute pain sensation and motor function.

Next, we tested pain behaviors after SNL. Consistent with previous reports (25), SNL induced persistent mechanical allodynia (Figure 5E) and heat hyperalgesia (Figure 5F) in WT mice. However, SNL-induced mechanical allodynia was significantly reduced in KO mice at all the time points we checked $(P<0.001,2$-way repeated measures ANOVA, Figure 5E). In addition, heat hyperalgesia did not develop in KO mice until 28 days $(P<0.001,2$-way repeated measures ANOVA, Figure 5F).

To further investigate the role of spinal CXCR5 in the maintenance of neuropathic pain, we intraspinally injected Cxcr 5 shRNA lentivirus (LV-Cxcr5 shRNA-3, Supplemental Table 1 and Supple- 
A

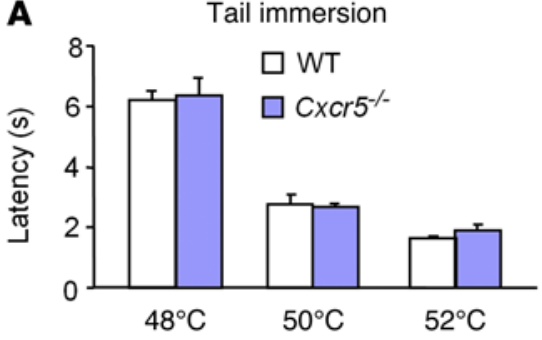

E

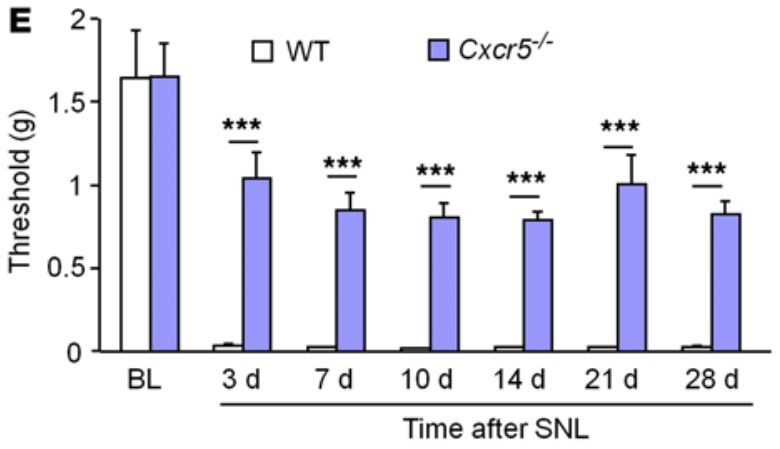

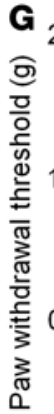

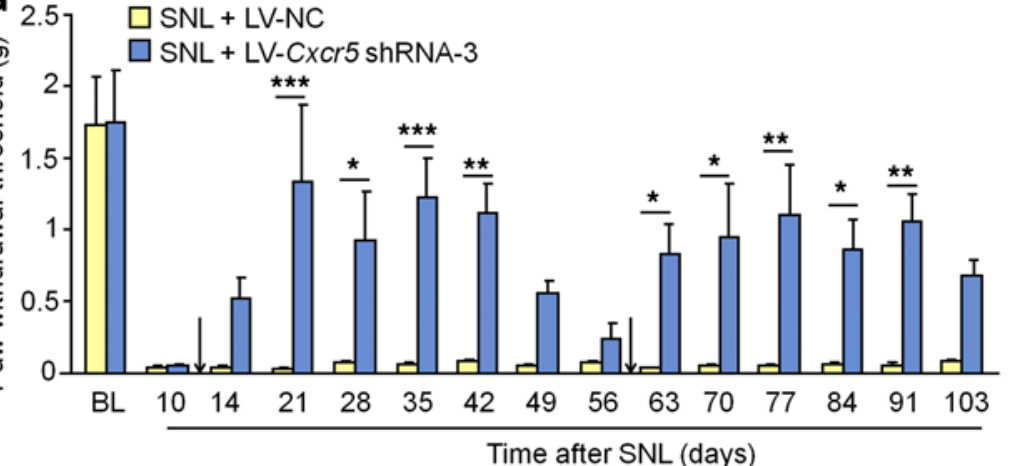

Time after SNL (days)

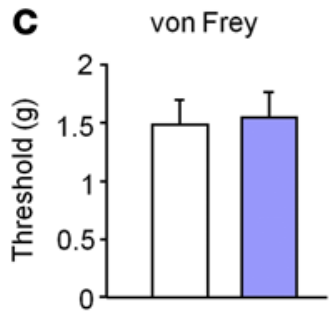

D Rotarod

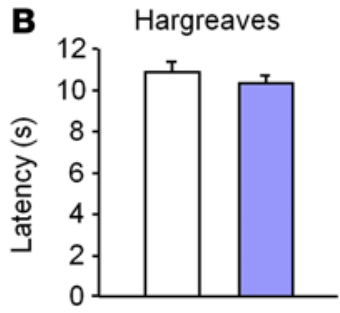

$\mathbf{F}$

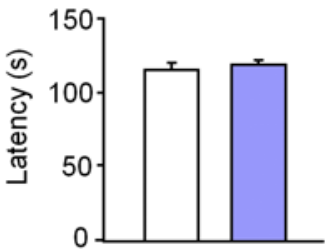

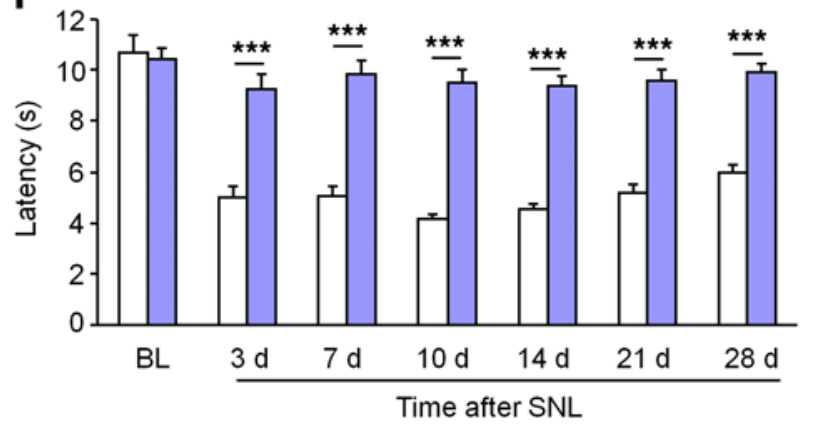

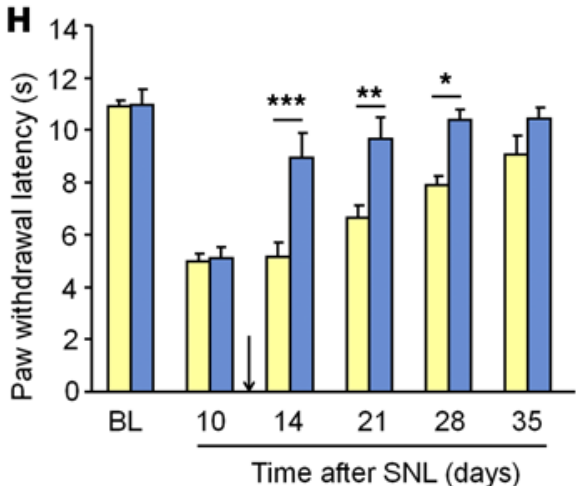

Figure 5. Cxcr5 is essential for SNL-induced neuropathic pain. (A-D) Acute pain thresholds and motor function are normal in Cxcr5 KO mice. Thermal sensitivity, measured by (A) tail immersion and (B) Hargreaves test, was comparable in WT and KO mice. Mechanical sensitivity assessed by von Frey test was indistinguishable in WT and KO mice (C). Motor function assessed by recording the falling latency in a Rotarod test was comparable in WT and KO mice (D). $n=11$ mice/group (A-C); $n=6$ mice/group (D). (E and F) SNL-induced mechanical allodynia (E) and heat hyperalgesia (F) were largely reduced in Cxcr5 KO mice $(n=10)$ compared with WT mice $(n=8)$. ${ }^{* *} P<0.001$, 2-way repeated measures ANOVA followed by Bonferroni's test. (G and $\left.\mathbf{H}\right)$ Intraspinal infusion of LV-Cxcr5 shRNA lentivirus in the spinal cord 10 days after SNL alleviated SNL-induced mechanical allodynia (G) and blocked heat hyperalgesia (H). ${ }^{*} P<0.05 ;{ }^{* *} P<0.01 ;{ }^{* *} P<0.001,2$-way repeated measures ANOVA followed by Bonferroni's test. $n=6$ mice/group.

mental Figure 13, A and B) and LV-NC 10 days after SNL in WT mice. LV-Cxcr5 shRNA-3 did not induce motor dysfunction or sedative behavior (Supplemental Figure 13, C and D). However, it attenuated SNL-induced mechanical allodynia from 21 to 42 days after SNL $(P<0.001,2$-way repeated measures ANOVA, Figure 5G). Notably, the second injection of LV-Cxcr5 shRNA still effectively attenuated mechanical allodynia in late-phase neuropathic pain (Figure 5G). LV-Cxcr5 shRNA-3 also completely reversed SNL-induced heat hyperalgesia $(P<0.001,2$-way repeated measures ANOVA, Figure $5 \mathrm{H}$ ), which was fully recovered at 35 days. qPCR data showed that LV-Cxcr5 shRNA reduced Cxcr5 mRNA expression by $70.5 \% \pm 5.8 \%$ compared with LV-NC 28 days after SNL $(P<0.01$, Student's $t$ test). In comparison, LV-Cxcr5-shRNA-2, which is less effective in knocking down CXCR5 expression than LV-Cxcr5-shRNA-3, produced only mild reduction in SNL-induced mechanical allodynia and heat hyperalgesia at some time points (Supplemental Figure 13, E and F).
Mice lacking Cxcr5 have defects in spinal glial activation after $S N L$. As CXCR5 was predominantly expressed in spinal astrocytes, we asked whether CXCL13/CXCR5 signaling contributes to astrocytic activation in the spinal cord after SNL. Immunostaining showed that basal GFAP IR was similar in naive WT (Figure 6A) and KO mice (Figure 6B). GFAP IR was significantly increased in the ipsilateral spinal cord at both day 7 $(P<0.001$, vs. sham, Student's $t$ test, Figure 6, C and M) and day 28 $(P<0.01$, vs. sham, Figure 6, E and M) after SNL in WT mice, but these increases were significantly reduced at day $7(P<0.05$, KO vs. WT, Figure $6, \mathrm{D}$ and $\mathrm{M})$ and day $28(P<0.01, \mathrm{KO}$ vs. WT, Figure 6, E and M) in Cxcr5 KO mice.

We also investigated microglial activation in WT and KO mice. Baseline IBA-1 IR was comparable in naive WT (Figure 6G) and $\mathrm{KO}$ (Figure $6 \mathrm{H}$ ) mice. SNL dramatically increased the expression of the microglial marker IBA-1 in the ipsilateral spinal cord at day $7(P<0.001$, vs. sham. Student's $t$ test, Figure 6, I and N) 
WT
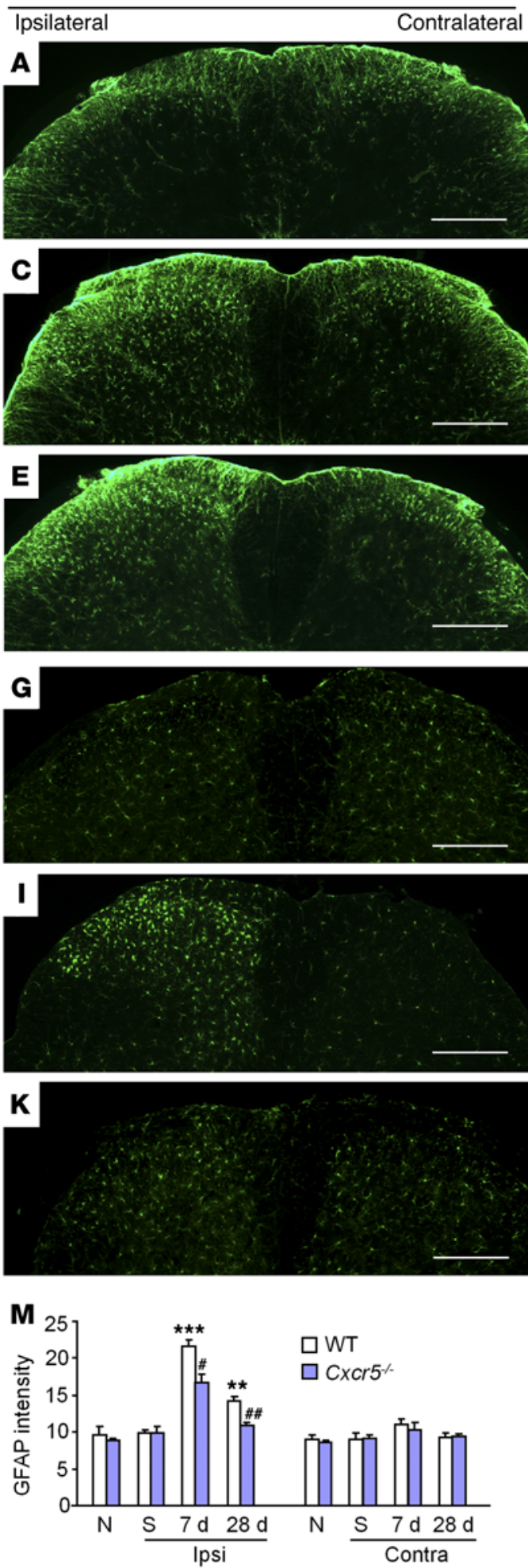

$\mathrm{CxCr5}^{-1-}$
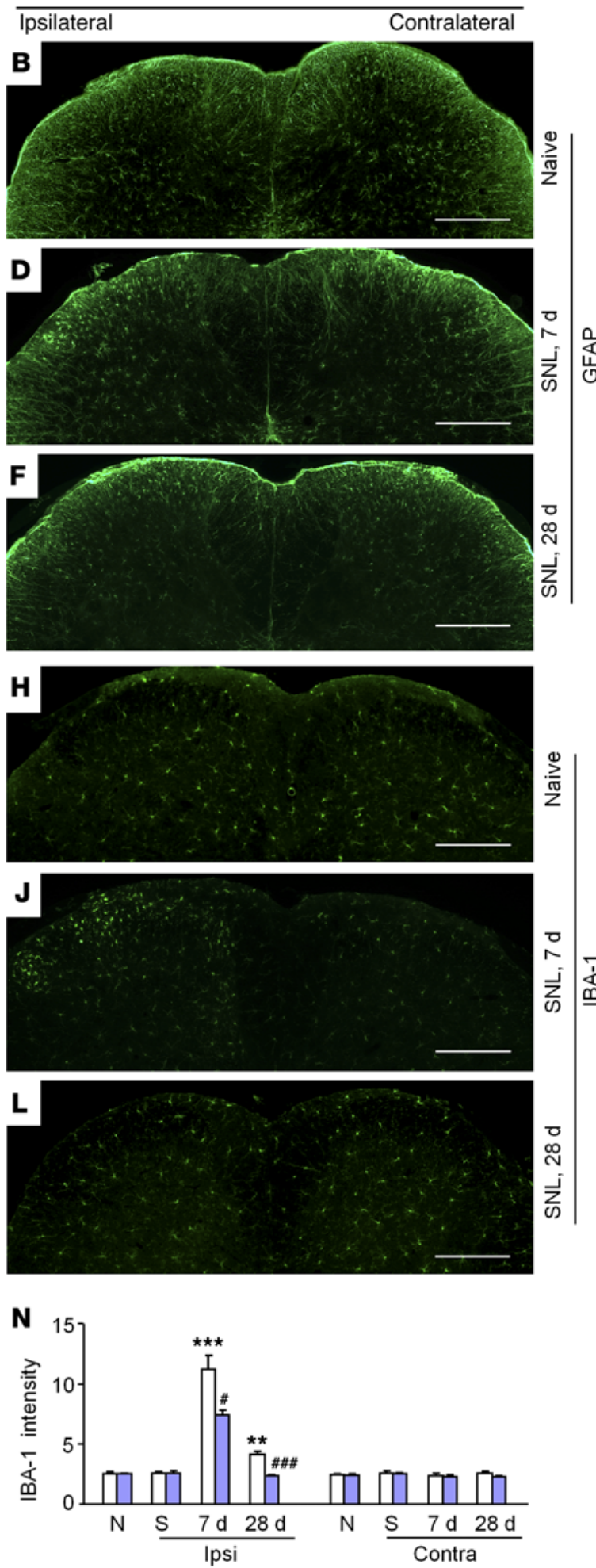

Figure 6. SNL-induced activation of glial cells in the spinal cord is inhibited in Cxcr5 KO mice. (A-F) GFAP staining in the spinal cord in naive (A and B), SNL at 7 days (C and $\mathbf{D})$, and SNL at 28 days (E and $\mathbf{F}$ ) of WT (A, C, and $\mathbf{E}$ ) and $C x C r 5$ KO (B, D, and $\mathbf{F}$ ) mice. (G-L) IBA-1 staining in the spinal cord in naive (G and $\mathbf{H}$ ), SNL at 7 days (I and J), and SNL at 28 days (K and $\mathbf{L}$ ) of WT (G, I, and $\mathbf{K}$ ) and Cxcr5 KO ( $\mathbf{H}$, J, and $\mathbf{L}$ ) mice. Scale bars: $200 \mu \mathrm{m}$. (M and $\mathbf{N}$ ) The intensity of GFAP (M) and IBA-1 (N) in the ipsilateral and contralateral spinal cord. ${ }^{* *} P<0.01$; ${ }^{* *} P<0.001$, SNL vs. sham, Student's $t$ test. ${ }^{*} P<0.05$; ${ }^{\# \#} P<0.01$; ${ }^{\# \#} P<0.001$, KO vs. WT, Student's $t$ test. N, naive. S, sham. $n=4$ mice/group. 
and day 28 ( $P<0.01$, vs. sham, Figure $5, \mathrm{~K}$ and $\mathrm{N})$ in WT mice. The nerve injury-induced activation of microglia (IBA-1 upregulation) was also significantly reduced in $\mathrm{KO}$ mice at both day 7 $(P<0.05, \mathrm{KO}$ vs. WT, Figure 6, J and N) and day $28(P<0.001$, KO vs. WT, Figure 6, L and N). These data indicate that CXCR5 plays an essential role for the activation of spinal astrocytes and microglia following SNL.

To further confirm the role of CXCR5 in distinct activation of microglia and astrocytes, we examined GFAP and IBA-1 expression in chemotherapy-induced neuropathy. It was shown that chemotherapy induced profound astrocyte activation, but limited microglial activation (31-33). Intraperitoneal injection of paclitaxel $(6 \mathrm{mg} / \mathrm{kg})$ induced mechanical allodynia, which started at 3 days and was maintained for more than 14 days in WT mice (Supplemental Figure 14A). However, paclitaxel-induced mechanical allodynia was significantly reduced in Cxcr5 KO mice $(P<0.05$ or 0.01 , vs. WT). Moreover, paclitaxel increased GFAP IR in WT mice but not in KO mice 7 days after injection (Supplemental Figure 14, B and D-G). However, IBA-1 IR was not changed in either WT or KO mice (Supplemental Figure 14, C and $\mathrm{H}-\mathrm{K})$. These data indicate that CXCR5 is important in mediating astrocytic activation in the spinal cord.

Spinal injection of CXCL13 induces CXCR5-dependent pain hypersensitivity and astrocytic activation in the dorsal horn. To investigate whether CXCL13 is sufficient to induce pain, we i.t. injected CXCL13 into WT and Cxcr5 KO mice. As shown in Figure 7A, CXCL13, but not vehicle (PBS), induced heat hyperalgesia in WT mice for more than 24 hours $(P<0.001,2$-way repeated measures ANOVA), whereas Cxcr5 KO mice failed to develop heat hyperalgesia. The i.t. CXCL13 also induced robust mechanical allodynia in WT mice, but this allodynia was reduced in $\mathrm{KO}$ mice (Figure 7B). These data indicate that CXCL13 is sufficient to induce pain hypersensitivity via its receptor CXCR5. Additionally, we cannot exclude that CXCL13 may also act on other receptors to induce mechanical allodynia, which needs further investigation in the future.

We then checked to determine whether CXCL13 would activate spinal glial cells after i.t. injection. CXCL13 induced significant and transient Gfap mRNA upregulation in the spinal cord at 6 hours $(P<0.01$, vs. control, Figure $7 C)$, but not at 1,3 , or 24 hours. In contrast, Iba1 mRNA was only increased at 24 hours $(P<0.001$, vs. control, Figure 7C). Immunostaining further showed that GFAP intensity was increased in the spinal cord dorsal horn 6 hours after CXCL13 injection in WT mice, but not in KO mice (Figure 7, D-H). To further determine whether CXCL13induced pain hypersensitivity is mediated by astrocytes and/or microglia, weinjected astroglial toxin L- $\alpha$-aminoadipate ( $L-\alpha-A A$, $10 \mathrm{nmol}$, i.t.) or microglial inhibitor minocycline (10 nmol, i.t.) (34) 1 hour prior to CXCL13 injection (100 ng, i.t.). Pretreatment with L- $\alpha$-AA blocked CXCL13-induced heat hyperalgesia at 1, 3, 6 , and 24 hours $(P<0.001,2$-way repeated measures ANOVA, Figure 7I). In contrast, minocycline attenuated the hyperalgesia at 24 hours $(P<0.05$, vs. vehicle, Figure $7 \mathrm{I})$. L- $\alpha$-AA also blocked CXCL13-induced mechanical allodynia (Figure 7J). However, minocycline had no effect on mechanical allodynia. We then checked Iba1 mRNA expression after behavioral testing and found that both minocycline and L- $\alpha$-AA pretreatment decreased CXCL13-induced Iba1 expression $(P<0.001$, 1-way ANOVA, Figure $7 \mathrm{~K})$. Given the earlier activation of astrocytes and later activation of microglia following spinal CXCL13 stimulation, these data suggest that spinal astrocytes are involved in initiating CXCL13-induced pain hypersensitivity.

CXCL13-induced astrocytic activation and pain hypersensitivity is dependent on ERK. As CXCL13-induced pain hypersensitivity began at 1 hour and CXCL13-induced Gfap increase was found 6 hours after injection, we asked whether there was an earlier event of astrocytic activation. It has been shown that ERK is activated in spinal glial cells after SNL and plays an important role in neuropathic pain (35). We first checked ERK activation via testing phosphorylated ERK (pERK) expression in the spinal cord after i.t. CXCL13. CXCL13 (100 ng) induced rapid pERK expression (1 hour) in the spinal dorsal horn in WT mice, but not in Cxcr5 KO mice (Figure 8, A and B). Additionally, double-immunofluorescence staining showed high colocalization of pERK with GFAP (Figure 8, C-F). Pretreatment with L- $\alpha$-AA (10 nmol, i.t.) inhibited CXCL13induced pERK expression 1 hour after CXCL13 injection (Figure $8 \mathrm{G})$, suggesting an astrocyte-dependent activation of ERK activation. Further, pretreatment with ERK kinase (MEK) inhibitor PD98059 (10 $\mu$ g, i.t.) (36) blocked CXCL13-induced heat hypersensitivity and mechanical allodynia $(P<0.001$ or 0.01 , 2-way repeated measures ANOVA, Figure $8, \mathrm{H}$ and I). The same treatment also reduced CXCL13-induced Gfap upregulation 6 hours after CXCL13 treatment (Figure 8J), suggesting that CXCL13induced astrocytic activation and pain hypersensitivity require CXCR5/ERK signaling.

We then checked pERK expression in the spinal cord after SNL. Immunostaining showed that pERK was colocalized with GFAP in the superfacial dorsal horn after SNL (Figure 8K), which is consistent with the reports from Zhuang and colleagues (35). Western blot showed that pERK was increased at both day 7 and day 28 after SNL in WT mice $(P<0.05$ or 0.001 , SNL vs. sham, Student's $t$ test). However, pERK expression was not significantly changed in Cxcr5 KO mice $(P<0.05$, SNL vs. sham, Figure $8 \mathrm{~L}$ ). These data suggest that SNL-induced ERK activation is CXCR5 dependent.

Injection i.t. of CXCL13-stimulated astrocytes induces mechanical allodynia. Our previous studies have shown that i.t. injection of TNF- $\alpha$-activated astrocytes can induce mechanical allodynia in naive mice $(37,38)$. To further confirm that CXCL13 enhances pain via causing activation of astrocytes, we cultured astrocytes from WT or $\mathrm{Cxcr5}^{-/}$mice, incubated these cells with CXCL13 (100 ng/ml) for 3 hours, and collected these WT and Cxcr5 $5^{-1-}$ astrocytes for i.t. injection into WT mice (Figure 9A). The i.t. injection of nontreated astrocytes decreased PWT in WT mice $(P<0.01$, 1-way repeated measures ANOVA, Figure 9B), suggesting that exogenous astrocytes can produce pronociceptive effects $(37,38)$. However, i.t. injection of CXCL13-treated WT, but not $\mathrm{CxCr}^{-/}$, astrocytes caused further reduction in PWT in WT mice compared with nontreated astrocytes (Figure 9B), indicating that CXCL13-activated astrocytes are sufficient to enhance pain states via CXCR5 activation in astrocytes.

We then determined whether CXCL13 is sufficient to activate ERK in cultured astrocytes. As shown in Figure 9C, following CXCL13 treatment, pERK expression was dramatically 

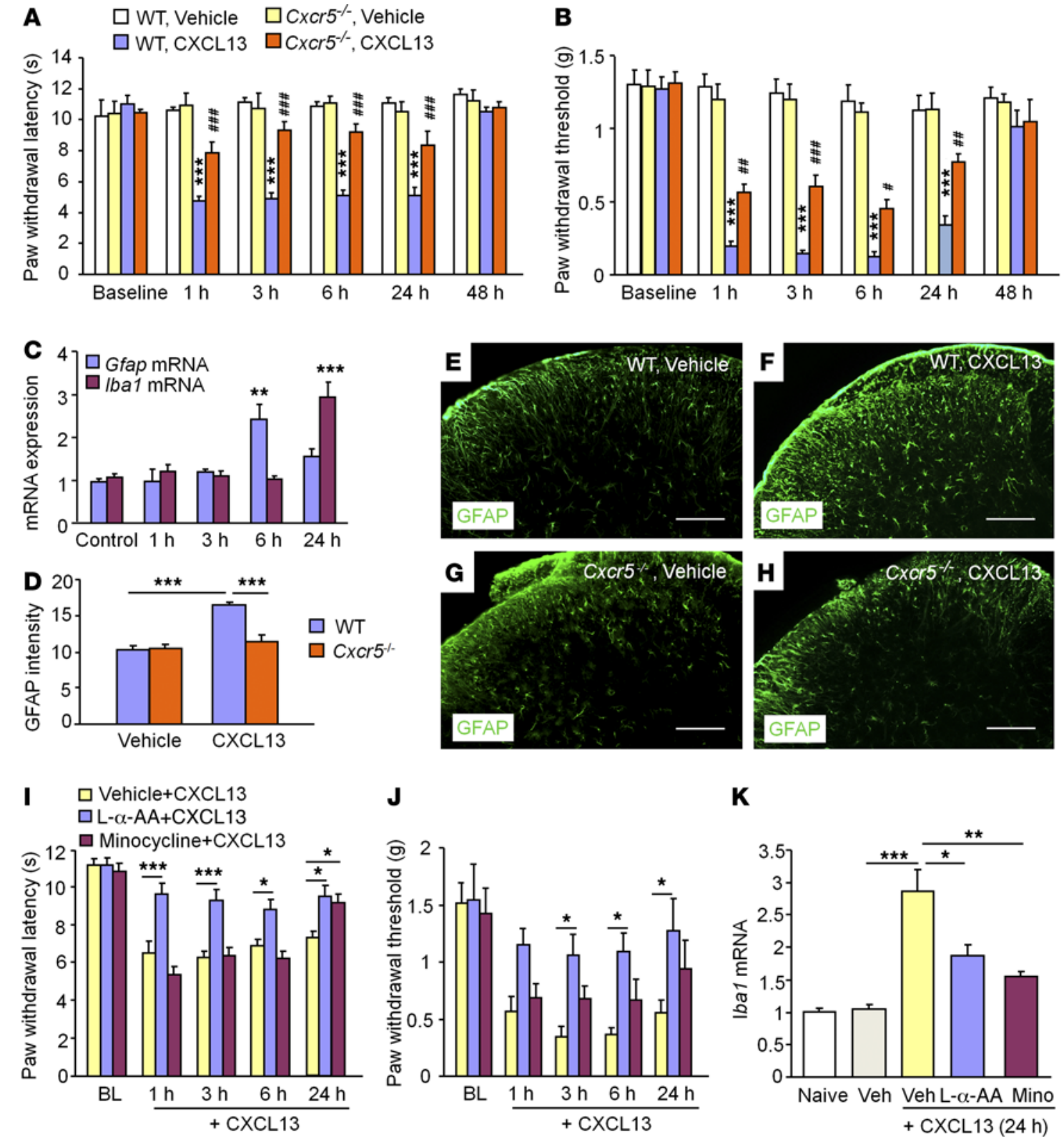

$\mathbf{K}$
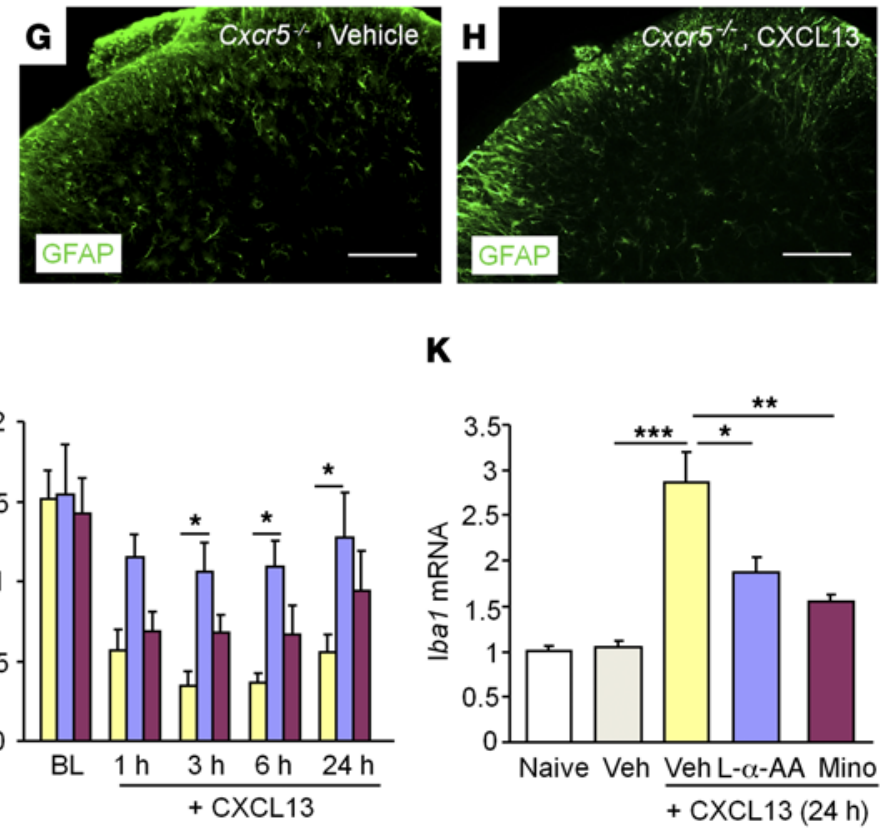

Figure 7. Injection (i.t.) of CXCL13 induces pain hypersensitivity and glial activation in the spinal cord. (A) Injection (i.t.) of CXCL13 induced heat hyperalgesia in WT mice, but not in Cxcr5 K0 mice. ${ }^{* * *} P<0.001$, WT vehicle $(n=7)$ vs. WT CXL13 $(n=7)$. \#\#\#< 0.001 , WT CXCL13 ( $\left.n=7\right)$ vs. Cxcr5 $5^{--}$ CXCL13 $(n=7)$, 2-way repeated measures ANOVA followed by Bonferroni's test. (B) Injection (i.t.) of CXCL13 induced mechanical allodynia in WT mice, which was reduced in KO mice. ${ }^{* *} P<0.001$, WT vehicle vs. WT CXL13. ${ }^{*} P<0.05 ;{ }^{\# \#} P<0.01 ;{ }^{\# \#} P<0.001$, WT-CXCL13 vs. CXCr5 $5^{-1-}$-CXCL13, $2-$ way repeated measures ANOVA followed by Bonferroni's test. (C) Injection (i.t.) of CXCL13 increased Gfap mRNA at 6 hours and Iba1 mRNA at 24 hours. ${ }^{* *} P<0.01$; ${ }^{* *} P<0.001$ vs. control, 1-way ANOVA followed by Bonferroni's test. $n=4-7$ mice/group. (D-H) Injection (i.t.) of CXCL13 increased GFAP IR in WT mice, but not in $C x c r 5$ KO mice 6 hours after injection. ${ }^{* *} P<0.001$, Student's $t$ test. $n=4$ mice/group. Scale bars: $100 \mu$ m. (I) Pretreatment with astroglial toxin L- $\alpha$-AA blocked CXCL13-induced heat hyperalgesia. Pretreatment with microglial inhibitor minocycline attenuated heat hyperalgesia at 24 hours. ${ }^{*} P<0.05$; ${ }^{* *} P<0.001$, 2-way repeated measures ANOVA followed by Bonferroni's test. $n=5$ mice/group. (J) Pretreatment with L- $\alpha$-AA inhibited CXCL13-induced mechanical allodynia. ${ }^{*} P<0.05,2$-way repeated measures ANOVA followed by Bonferroni's test. $n=5$-8 mice/ group. (K) L- $\alpha-A A$ and minocycline decreased CXCL13-induced Iba1 mRNA upregulation in the spinal cord 24 hours after CXCL13 injection. ${ }^{*} P<0.05$; ${ }^{* *} P<0.01 ;{ }^{* *} P<0.001,1$-way ANOVA followed by Bonferroni's test. $n=3-6$ mice/group. Veh, vehicle.

increased at 30 minutes and maintained for more than 1 hour. However, CXCL13 failed to induce pERK expression in astrocytes from $\mathrm{Cxcr5} 5^{-/-}$mice, suggesting that ERK activation by CXCL13 also requires CXCR5 in astrocytes in vitro.

CXCL13 and CXCR5 are expressed in human spinal cord. Finally, we checked the expression of Cxcl13 and Cxcr5 in human tissues of spinal cord, DRG, and lymph node from nondiseased donors. PCR showed that both Cxcl13 and Cxcr5 mRNAs were highly expressed in lymph node, with low expression in the DRG and spinal cord (Supplemental Figure 15, A and B). Moreover, CXCL13 and CXCR5 protein were also detectable in the spinal cord by ELISA and Western blot, although the levels were much lower than those of lymph node 
A

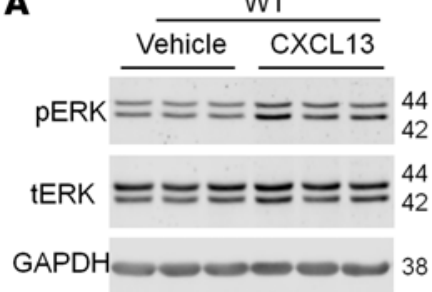

B

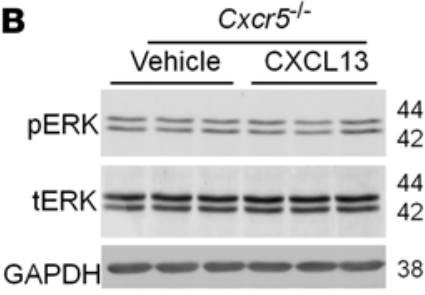

G

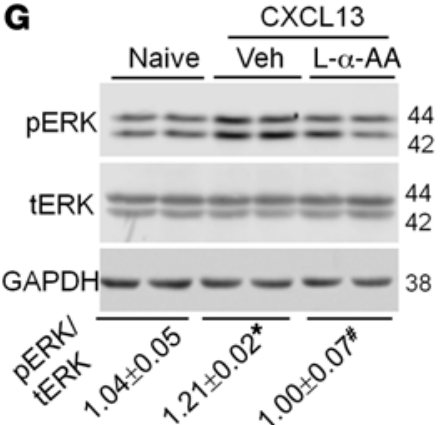

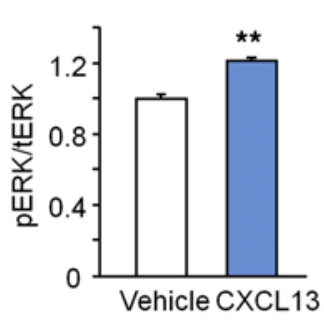
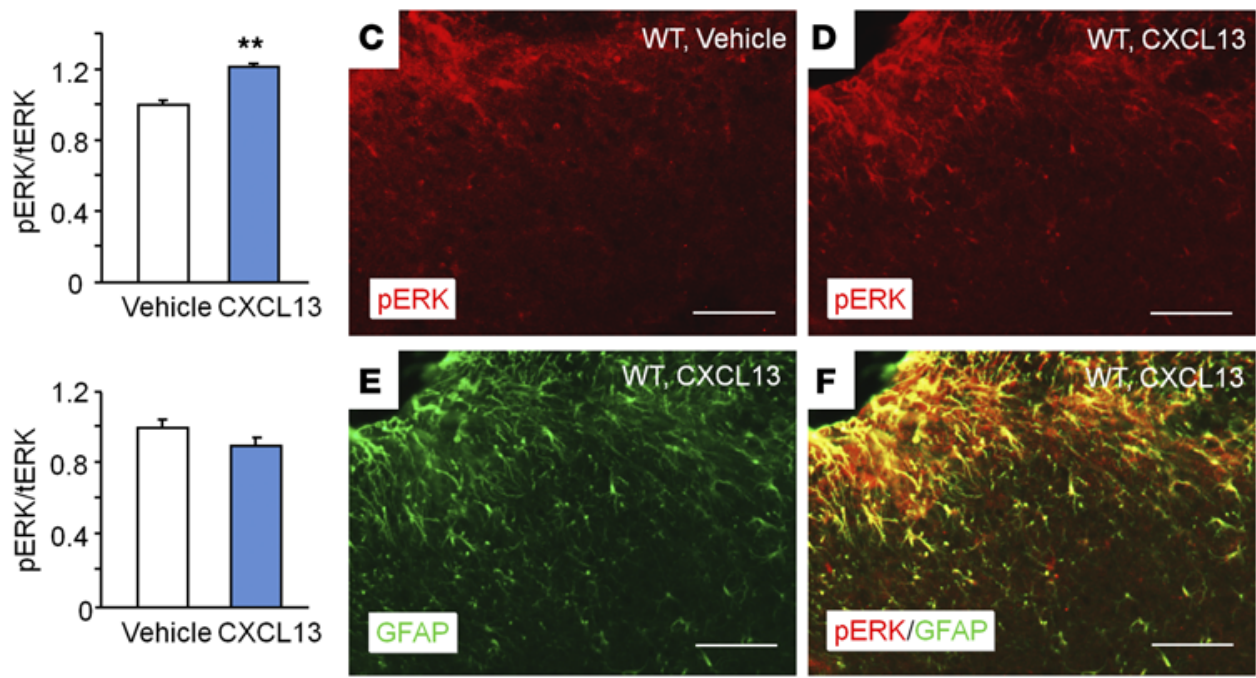

H

$\square$ Vehicle + CXCL13

$\square$ PD98059 + CXCL13

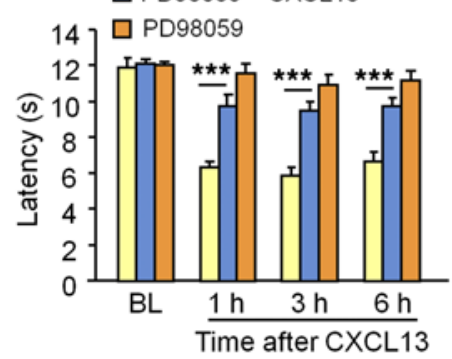

I

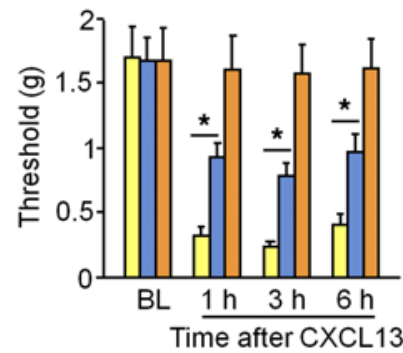

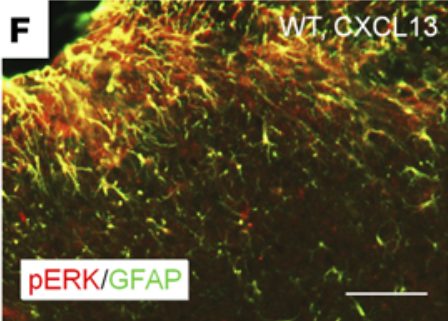

J

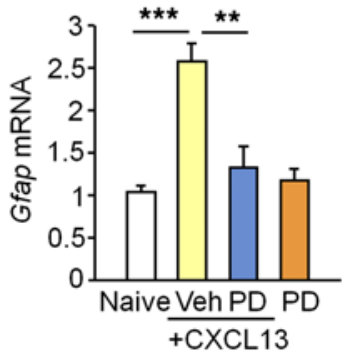

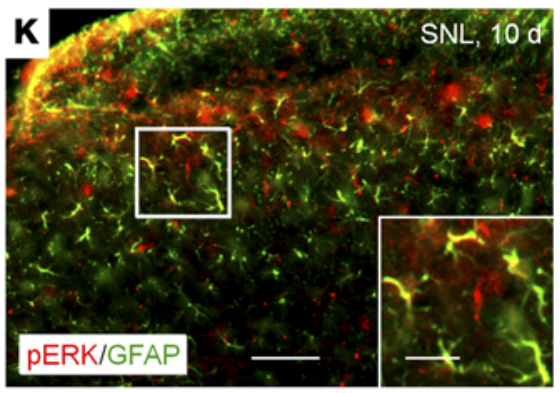
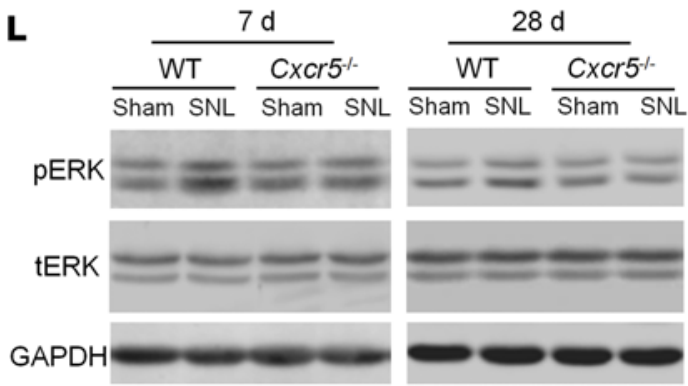

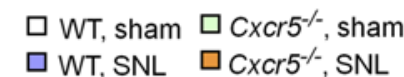

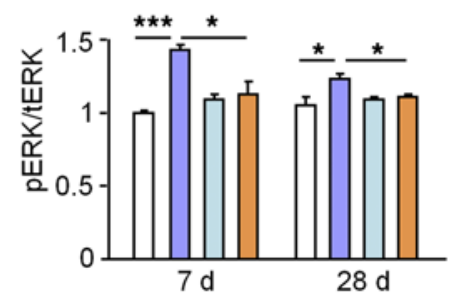

Figure 8. CXCL13 induces ERK-dependent astrocytic activation and pain hypersensitivity. (A) Injection (i.t.) of CXCL13 increased pERK expression in the spinal cord in WT mice (A), but not in CXCr5 KO mice (B). ${ }^{* *} P<0.01$, Student's $t$ test. $n=3$ mice/group. (C-F) Injection (i.t.) of CXCL13 increased pERK IR compared with i.t. vehicle (PBS) (C and D). pERK (D) was colocalized with GFAP (E and F) in the spinal cord 1 hour after injection. Scale bars: $50 \mu \mathrm{m}$. (G) Pretreatment with L- $\alpha$-AA inhibited CXCL13-induced pERK expression in the spinal cord 1 hour after CXCL13 injection. ${ }^{*} P<0.05$, vs. naive. ${ }^{*} P<0.05$, vs. veh + CXCL13, Student's $t$ test. $n=4$ mice/group. (H and I) Pretreatment with MEK inhibitor PD98059 blocked CXCL13-induced heat hyperalgesia $(\mathbf{H})$ and mechanical allodynia $(\mathbf{I}) .{ }^{*} P<0.05 ;{ }^{* * *} P<0.001,2$-way repeated measures ANOVA followed by Bonferroni's test. $n=6$ mice/group. (J) PD98059 inhibited CXCL13-induced Gfap mRNA increase at 6 hours. ${ }^{* *} P<0.01$; ${ }^{* *} P<0.001,1$-way ANOVA followed by Bonferroni's test. $n=3-5$ mice/group. (K) Colocalization of pERK and GFAP in the spinal cord 10 days after SNL. Scale bars: $50 \mu \mathrm{m} ; 20 \mu \mathrm{m}$ (insets). (L) pERK expression was increased in the spinal cord at 7 days and 28 days after SNL in WT mice, but not in $C x C r 5 \mathrm{KO}$ mice. ${ }^{*} P<0.05$; ${ }^{* * *} P<0.001$, Student's $t$ test. $n=3$ mice/group.

(Supplemental Figure 15, C and D). These data suggest the possible involvement of CXCL13 signaling in human pain conditions.

\section{Discussion}

CXCL13 was originally classified into a lymphoid chemokine, as it plays an essential role in the establishment and maintenance of lymphoid tissue microarchitecture (14). Over the past years, it has become evident that, in addition to its role in peripheral lymphoid tissue, CXCL13 is also implicated in neuroinflammation, particularly in autoimmune demyelination $(16,20)$ and neuroborreliosis $(39,40)$. In the present study, we reveal, for what we believe is the first time, the role and mechanism of CXCL13 and its receptor CXCR5 in neuropathic pain. We have made the following findings. First, CXCL13 was dramatically and persistently upregulated in spinal neurons following SNL and negatively regulated by miR-186-5p, whereas CXCR5 was increased in spinal 


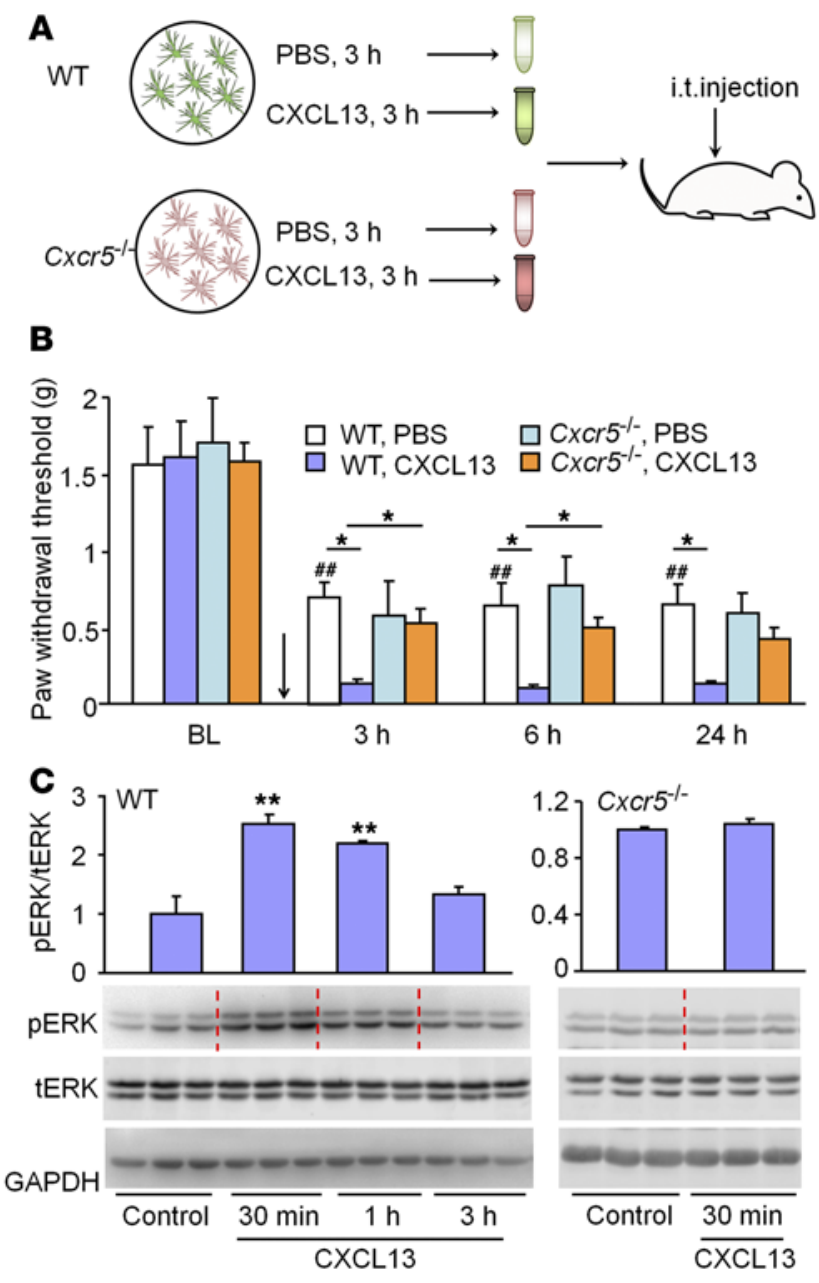

astrocytes. Second, spinal inhibition of CXCL13 or CXCR5 persistently attenuated SNL-induced pain hypersensitivity. Deletion of CXCR5 not only attenuated neuropathic pain, but also greatly reduced the activation of glial cells and ERK in the spinal cord. Third, i.t. injection of CXCL13 induced CXCR5/ERK-dependent pain hypersensitivity. CXCL13 also induced rapid astrocytic activation and delayed microglial activation. Taken together, our results indicate that CXCL13/CXCR5 drives neuropathic pain via neuronal-astrocytic interaction in the spinal cord.

CXCL13 upregulation in spinal neurons contributes to neuropathic pain. Neuroinflammation has been recently recognized for its pivotal role in the pathogenesis of neuropathic pain $(5,41,42)$. Mounting evidence suggests that chemokines, which comprise more than 50 family members, play an important role in mediating neuroinflammation $(4,42)$. In particular, several chemokines, including CCL2, CCL7, and CXCL1, are upregulated in the spinal cord following nerve injury and contribute to neuropathic pain (6, $7,10,11)$. In the present study, both microarray and qPCR data support the upregulations of these chemokines. ELISA and immunostaining further showed the increase of CXCL13 protein after SNL. Interestingly, CCL2, CCL7, and CXCL1 are expressed in spinal astrocytes $(6,7,10)$. Previous studies have shown that CXCL13 was induced in some microglia, macrophages, and endothelial cells in the CNS after infection $(40,43)$ or in infiltrating dendritic
Figure 9. Injection (i.t.) of CXCL13-treated astrocytes induces mechanical allodynia. (A) Experimental protocol showing the preparation of astrocytes for i.t. injection. The differentiated astrocytes from WT or $\mathrm{CXCr}^{-1-}$ mice were incubated with PBS or CXCL13, then collected, washed with PBS, and resuspended for i.t. injection into naive mice. (B) Injection (i.t.) of CXCL13-treated astrocytes from WT animals $(n=7)$ induced dramatic decrease in PWT, and this decrease was compromised after injection of $C x c r 5$-deficient astrocytes $(n=9)$. $\# P<0.01$ vs. baseline, 1-way repeated measures ANOVA followed by Bonferroni's test; ${ }^{*} P<0.05$, 2-way repeated measures ANOVA followed by Bonferroni's test. (C) CXCL13 induced rapid pERK expression in astrocyte cultures from WT, but not from $C x \mathrm{Cr} 5 \mathrm{KO}$ mice. ${ }^{*} P<0.01$ vs. control, 1-way ANOVA followed by Bonferroni's test. $n=3$ for each treatment.

cells in EAE mice $(16,17)$. Our present data show that CXCL13 was predominantly produced by spinal neurons after SNL. In cultured dorsal horn neurons, CXCL13 was localized in soma and neurites and densely packed in vesicles, indicating possible release of CXCL13 from neurons. Our behavioral data further show that inhibition of CXCL13 by shRNA lentivirus persistently attenuated SNL-induced mechanical allodynia and heat hyperalgesia, suggesting an important role of spinal CXCL13 in neuropathic pain.

miR-186-5p regulates CXCL13 expression in neuropathic pain. miRNAs are shown to posttranscriptionally regulate gene expression and play important roles in regulating immune responses, inflammation, and chronic pain $(44,45)$. In this study, although several miRNAs target the $3^{\prime}$ UTR of CXCL13 mRNA, only the expression of miR-186-5p was negatively correlated with the expression of CXCL13 mRNA following SNL. Immunostaining showed that miR-186-5p was colocalized with CXCL13 in spinal cord neurons. Moreover, overexpression of spinal miR-186-5p decreased CXCL13 expression and attenuated SNL-induced pain hypersensitivity. In contrast, inhibition of miR-186-5p was sufficient to increase CXCL13 expression and induce pain hypersensitivity. Interestingly, both SNL-induced miR-186-5p downregulation and CXCL13 upregulation were mediated by NMDAR, a key player in the pathogenesis of neuropathic pain (28). Our results demonstrate that miR-186-5p is involved in neuropathic pain via the regulation of CXCL13 expression.

Each miRNA is thought to control hundreds of target genes. Although the known targets of miR-186-5p are still limited, it has been reported that miR-186 targets cyclin D1, cyclin-dependent kinase 2 (CDK2), and CDK6 in non-small cell lung carcinoma cells (46). miR-186, in cooperation with miR-150, also downregulates expression of the purinergic $\mathrm{P} 2 \mathrm{X} 7$ receptor in cancer epithelial cells (27). Given the important role of spinal P2X7 receptor in the pathogenesis of neuropathic pain $(47,48)$, miR-186-5p may also contribute to neuropathic pain by regulating the $\mathrm{P} 2 \mathrm{X} 7$ receptor. Moreover, it was more recently reported that miR-186-5p was downregulated in the whole blood of patients suffering from oral squamous cell carcinoma (49) and was also downregulated in clinical lung adenocarcinoma specimens, and its downregulation correlates with poor survival in lung asemocarcinoma (46). These data indicate the potential development of miR-186-5pbased therapeutic approaches for treating certain types of cancer as well as neuropathic pain.

CXCR5-ERK cascade contributes to neuropathic pain. The chemokines exert their biological effects via G-protein-coupled 

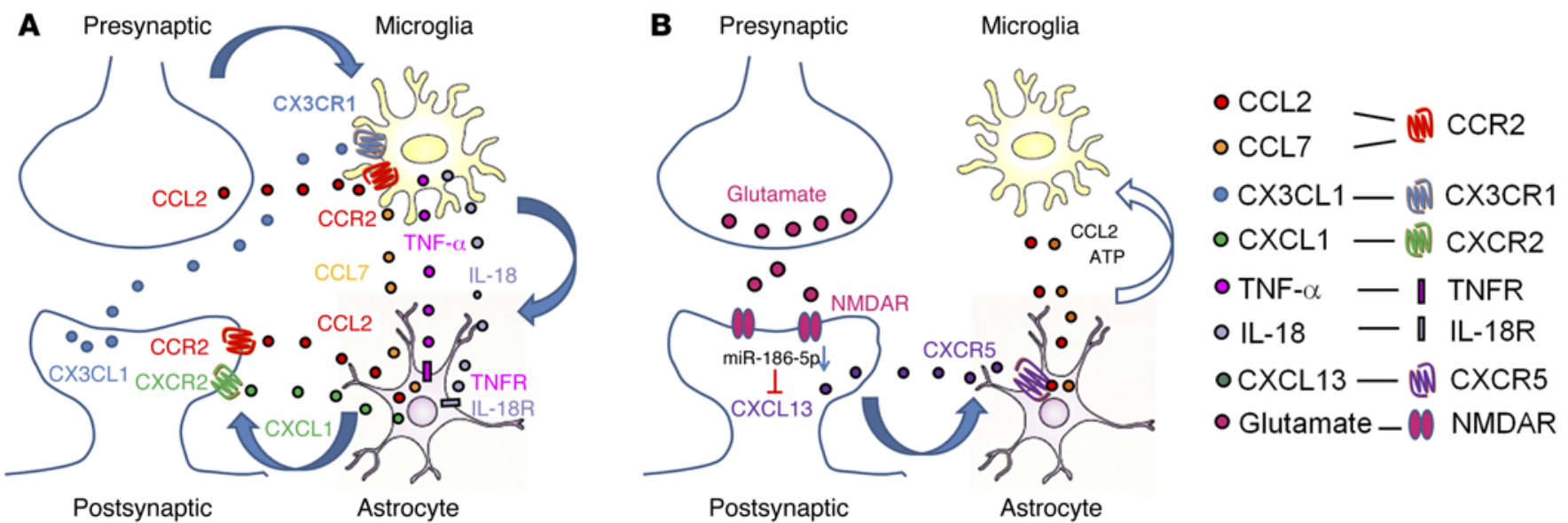

Figure 10. Schematic shows spinal neuronal-glial interactions in neuropathic pain after peripheral nerve injury. (A) Neuronal-glial interactions revealed in previous studies. After nerve injury, CCL2 and CX3CL1 are released from the central terminals of primary afferent neurons (presynaptic sites) and spinal neurons (postsynaptic sites), which activate CCR2 and CX3CR1, respectively, on spinal microglia. CCL2 and CXCL1 can be released from astrocytes to act on spinal neurons via CCR2 and CXCR2, respectively. Astrocytes may also produce CCL7 to recognize CCR2 on microglia. Upon activation, microglia produce cytokines TNF- $\alpha$ and IL-18 to activate astrocytes via TNFR and IL-18R. Microglia also produce the neurotrophin brainderived neurotrophic factor (BDNF) via P2X4 activation to enhance nociceptive synaptic transmission (63, 68). (B) Neuronal-glial interactions revealed in the present study. SNL increases glutamate release from presynaptic terminals to activate NMDAR on postsynaptic neurons, leading to decreased expression of miR-186-5p, and this decrease results in CXCL13 upregulation in spinal neurons. Upon release, CXCL13 acts on CXCR5 in astrocytes and activates the ERK signaling pathway, leading to the expression and release of astroglial mediators, such as CCL2 and CCL7, to maintain microglial activation. Astrocytes may also produce ATP to activate microglia via P2X4, P2X7, and P2Y12 receptors (47, 63, 69). These astrocytic mediators can also directly potentiate nociceptive transmission via pre- and postsynaptic modulation (10, 52). Together, this CXCL13-CXCR5-mediated neuronal-astrocytic interaction in the dorsal horn can enhance and prolong neuropathic pain states.

chemokine receptors. Although most chemokines activate multiple receptors CXCL13 and CXCR5 were thought to represent a monogamous chemokine/chemokine receptor pair (1). Previous studies showed that CXCL13 regulates homing or recruiting of $\mathrm{B}$ cells via CXCR5 in the immune system and the CNS $(14,15)$. Here, we demonstrated a new role of CXCR5 in mediating neuropathic pain in the spinal cord. Cxcr5 KO mice showed marked deficits in SNL-induced heat hyperalgesia and mechanical allodynia. Consistently, knockdown of CXCR5 in the spinal cord by shRNA lentivirus reversed SNL-induced pain hypersensitivity in WT mice for more than 4 weeks. Furthermore, the second LV-Cxcr 5 shRNA injection was still effective in reversing late-phase neuropathic pain. These data suggest that CXCR5 is necessary for the maintenance of neuropathic pain. Moreover, i.t. injection of CXCL13 induced CXCR5-dependent heat hyperalgesia and mechanical allodynia for more than 24 hours, indicating that activation of CXCR5 is also sufficient to induce pain hypersensitivity.

ERK is important for chronic pain sensitization, and i.t. injection of selective inhibitors of MEK was shown to attenuate inflammatory, neuropathic, and cancer pain in rats and mice (50). pERK is expressed in both microglia and astrocytes in the spinal cord in chronic pain conditions (41). Specifically, pERK induction was observed in spinal microglia in the early phase (first week), but found in astrocytes in the late phase after nerve injury (35). Here, we show that in Cxcr5 KO mice, ERK activation was inhibited in the spinal cord in both the early phase (7 days) and the late phase (28 days) of neuropathic pain. ERK was also activated in the spinal cord by i.t. injection of CXCL13 or in astrocyte cultures by incubation with CXCL13. Moreover, MEK inhibitor PD98059 blocked CXCL13-induced pain hypersensitivity and Gfap upregulation. These results demonstrated that ERK is a downstream event of CXCL13/CXCR5 signaling. Research shows that ERK in astrocytes may mediate the expression of inflammatory mediators, including proinflammatory cytokines (e.g., IL-1ß) and chemokines (e.g., CCL2, CXCL1) $(7,10,51)$. It is likely that the activation of ERK in astrocytes by CXCL13 may induce the release of inflammatory mediators to modulate central sensitization and chronic pain $(7,10,52)$.

A form of neuron-glial interaction in neuropathic pain mediated by CXCL13-CXCR5. Previous studies have implicated several chemokines in neuronal-microglial interaction in neuropathic pain. For example, chemokines CX3CL1 and CCL21 are expressed in primary sensory neurons and induce microglial activation via their microglial receptors CX3CR1 and CCR7/ CXCR3, respectively $(9,12,53,54)$. Our previous studies have also demonstrated astroglial-neuronal interaction in neuropathic pain via respective expression of CCL2 and CXCL1 in spinal astrocytes and CCR2 and CXCR2 in spinal neurons; and furthermore, direct activation of CCR 2 and CXCR2 by these chemokines rapidly, within minutes, enhances spinal cord nociceptive synaptic transmission (refs. 7, 10, and Figure 10A). In this study, we provided several lines of evidence to demonstrate a previously unrecognized CXCL13-CXCR5-mediated neuronal-astroglial interaction that is critically important for persistent neuropathic pain (Figure 10B). First, spinal astrocytic activation was abrogated in Cxcr5 KO mice after SNL- or paclitaxel-induced neuropathy. Second, i.t. injection of CXCL13 induced marked activation of astrocytes, which was abolished in Cxcr5 KO mice. Third, CXCL13-induced pain hypersensitivity was blocked by pretreatment with astroglial toxin. Fourth, i.t. injection of 
CXCL13 induced ERK activation in spinal astrocytes, which is required for CXCL13-induced pain hypersensitivity. Finally, i.t. injection of CXCL13-treated WT astrocytes, but not Cxcr5deficient astrocytes, induced mechanical allodynia in naive mice. In addition to astrocytes, CXCR5 in spinal cord neurons may also play a role in neuropathic pain, since a small number of spinal cord neurons also express CXCR5.

Accumulating evidence suggests that in inflammatory pain and neuropathic pain conditions, microglial reaction (upregulation of IBA-1 and CD11b and microgliosis) precedes astrocytic reaction (upregulation of GFAP and astrogliosis) and further causes astrocytic reaction (55-57). Indeed, nerve injury increased the expression of IL-18 and TNF- $\alpha$ in reactive microglia, which act on astrocytes via their respective receptors IL-18R and TNFR and enhance neuropathic pain states (refs. 37, 58, 59, and Figure 10A). In contrast, our data suggest that astrocytes may also drive microglial activation. Although CXCR5 is not expressed in spinal microglia, both astrocytic and microglial activation were reduced in Cxcr5 KO mice after SNL. Moreover, spinal injection of CXCL13 not only induced rapid astrocytic activation at 6 hours, but also induced delayed microglial activation at 24 hours (Figure 7C). Importantly, inhibition of astrocyte function also inhibited microglial activation. Activated astrocytes may release $\operatorname{CCL} 2(6,10)$ to activate microglia, since its receptor CCR2 might be expressed in microglia $(60,61)$ in addition to neurons (Figure 10A). Astrocytes can also release ATP (62) to induce microglial activation and proliferation via $\mathrm{P} 2 \mathrm{X} 7$ and $\mathrm{P} 2 \mathrm{X} 4$ receptors $(47,63$, $64)$. Although primary sensory neurons are well known as driving microglia activation after nerve injury, astrocytes may play a unique role in maintaining microglial activation in neuropathic pain by releasing ATP and CCL2.

In conclusion, our results demonstrate that peripheral nerve injury induces robust neuronal expression of chemokine CXCL13, which can be released from spinal neurons, acts on its receptor CXCR5 in astrocytes, and induces astrocytic activation for the maintenance of neuropathic pain. CXCL13/CXCR5 is the only chemokine pair that can mediate spinal neuronal-astrocytic interaction in neuropathic pain. Interestingly, CXCL13 is negatively regulated by miR-186-5p, and its downregulation in spinal neurons after SNL causes upregulation of CXCL13 to drive neuropathic pain. Thus, strategies that target CXCL13/ CXCR5 signaling via inducing miR-186-5p expression or blocking CXCR5 function may lead to novel therapies for the treatment of neuropathic pain.

\section{Methods}

Additional details regarding this study are provided in Supplemental Methods.

Animals, surgery, and human tissues. Adult ICR mice and C57BL/6 mice were purchased from the Experimental Animal Center of Nantong University. CXCR5-deficient mice (B6.129S2[Cg]-Cxcr5 $5^{\text {tmlLipp/J })}$ were purchased from the Jackson Laboratory. The L5 SNL was produced as previously described (65). Human spinal cords and DRG tissues were obtained from nondiseased donors through the National Disease Research Interchange (NDRI). Healthy human lymph nodes were provided by the Department of Pathology of the Affiliated Hospital of Nantong University.
Drugs. CXCL13 was purchased from PeproTech. miR-186-5p mimic, miR-186-5p inhibitor, and their scrambled NC were synthesized by RiboBio.

Lentiviral vector production and intraspinal injection. Three shRNAs targeting murine Cxcl13 (LV-Cxcl13 shRNA) or Cxcr5 (LV-Cxcr5 shRNA) were designed. The pre-mmu-miR-186 sequence was inserted into lentiviral vector pGV209, which regulates the expression of pre-miRNAs by an $\mathrm{H} 1$ promoter, and was then packaged into lentivirus. The sequences are shown in Supplemental Table 1. The intraspinal injection was performed as described previously (7).

RNA isolation, microarray, and bioinformatics analysis. Total RNA was isolated from the L5 spinal cord using the TRIzol reagent (Invitrogen). Gene-expression profiles of the spinal cord were assessed with Agilent SurePrint G3 Mouse GE 8×60K Microarray Kit (G4852A; CapitalBio Corp.). All original microarray data were deposited in the NCBI's Gene Expression Omnibus (GEO GSE75072).

The conserved miRNA binding sites of Cxcl13 were predicted by TargetScan and are shown in Supplemental Table 2.

Real-time qPCR. Total RNA was extracted using Trizol reagent and was reverse transcribed using an oligo (dT) primer. qPCR analysis was performed with the Real-Time Detection System by SYBR green I dye detection (Takara). Detailed primer sequences for each gene are listed in Supplemental Table 3.

Semiquantitative reverse-transcription PCR (RT-PCR). After the RNA extraction and CDNA reverse transcription, $c D N A$ samples were amplified. The primer sequences for human genes are listed in Supplemental Table 4 . The PCR products were electrophoresed on agarose gel and visualized under UV light.

Construction of vectors and dual-luciferase reporter assays. The partial 3' UTR sequences of Cxcl13 that contain the miRNAs' target region or mutant region were cloned into the pmirGLO Dual-Luciferase Reporter Vector (Promega). The oligonucleotide sequences used for producing matched and mismatched miRNA binding site regions are shown in Supplemental Table 5. Activities of firefly and Renilla luciferase were measured using the Dual-Glo Luciferase Assay System (Promega).

Dorsal horn neurons culture. Primary cultures of spinal cord dorsal horn neurons were prepared from neonate ICR mice (P3) using a procedure modified from a previously described method (66).

Astrocyte culture. Primary astrocyte cultures were prepared from cerebral cortexes of neonatal WT or Cxcr5 KO mice (P2) (10). The cerebral hemispheres were isolated, minced, filtered, and cultured in low-glucose DMEM. The cells were incubated with CXCL13 and collected for Western blot or i.t. injection.

ELISA. The Mouse CXCL13 ELISA Kit was purchased from R\&D Systems. The tissues were homogenized in a lysis buffer containing protease and phosphatase inhibitors (Sigma-Aldrich). ELISA was performed according to the manufacturer's protocol.

Western blot. Protein samples were prepared in the same way as for ELISA analysis. Protein samples were separated on SDS-PAGE gel and transferred to nitrocellulose blots. The blots were blocked and incubated overnight at $4^{\circ} \mathrm{C}$ with antibodies against CXCR5 (rabbit, 1:100, Santa Cruz Biotechnology Inc., sc-30029), pERK (rabbit, 1:500, Cell Signaling, 9101), ERK (rabbit, 1:500, Cell Signaling, 9102), and GAPDH (mouse, 1:20000, Millipore, MAB374).

Immunohistochemistry and immunocytochemistry. Immunohistochemistry was performed according to standard protocols, and the following primary antibodies were used: CXCL13 (goat, 1:100, Santa 
Cruz Biotechnology Inc., sc-8182) or CXCR5 (rabbit, 1:100; Santa Cruz Biotechnology Inc, sc-30029), GFAP (mouse, 1:5000, Millipore, MAB360), NeuN (mouse, 1:1000, Millipore, MAB377), IBA-1 (rabbit, 1:3000, Wako, 019-19741), OX-42 (mouse, 1:100, AbD Serotec, MCA275GA), and pERK (rabbit, 1:500, Cell Signaling, 9101). Immunocytochemistry was performed on cultured dorsal horn neurons, which were fixed with $4 \%$ paraformaldehyde and processed for immunofluorescence with CXCL13 (goat, 1:200; Santa Cruz Biotechnology Inc., sc-8182) and MAP2 (mouse, 1:5000, Sigma-Aldrich, M1406).

In situ hybridization for CXCL13 mRNA and miR-186-5p. Cellular localization of CXCL13 was performed using the mouse Cxcl13 mRNA ISH Assay Kit (Boster) (7). Locked nucleic acid-based in situ detection of miRNA-186 was performed as described previously (67). To identify the cell types expressing CXCL13 and miR-186-5p, the above sections under ISH were incubated overnight at $4^{\circ} \mathrm{C}$ with primary antibodies against GFAP, NeuN, IBA-1, or CXCL13 for double staining.

Behavioral analysis. Animals were habituated to the testing environment daily for at least 2 days before baseline testing. Acute thermal sensitivity was assessed by radiant heat and hot water immersion. Heat hyperalgesia and mechanical allodynia were performed as previously described $(7,10)$. Motor function was tested by Rotarod test. The sedative potential of drugs was investigated by recording spontaneous locomotor activity of mice in an open-field test. The behaviors were tested blindly.

Statistics. All data were expressed as mean \pm SEM. Differences between groups were compared using 1-way ANOVA or 2-way repeated measures ANOVA followed by Bonferroni's test or by Student's $t$ test (2-tailed) if only 2 groups were applied. Correlation analysis was assessed by Pearson's correlation test. The criterion for statistical significance was $P<0.05$.
Study approval. All animal procedures performed in this study were reviewed and approved by the Animal Care and Use Committee of Nantong University and were performed in accordance with the guidelines of the International Association for the Study of Pain.

\section{Author contributions}

BCJ analyzed the array data, designed the primers, and performed in situ hybridization, PCR, ELISA, and dual-luciferase reporter assay. DLC carried out cell culture, immunostaining, Western blot, and behavioral experiments. XZ and ZJZ performed immunostaining, PCR, and behavioral testing. BCJ and DLC analyzed the data. LNH, CHL, WWZ, and XBW participated in Western blot, PCR, and behavioral testing. TB performed PCR of human samples under the guidance of RRJ. YJG conceived the project and coordinated and supervised the experiments. YJG, BCJ, and RRJ wrote the paper.

\section{Acknowledgments}

This study was supported by the National Natural Science Foundation of China (NSFC 31371121, 31171062, 81300954, 81400915, and 81571070), the National Science Foundation for Young Scientists of Jiangsu Province (BK20140427), the Natural Science Research Program of Jiangsu Province (13KJB180016 and 13KJB180017), the Priority Academic Program Development of Jiangsu Higher Education Institutions, and NIH grants R01 DE17794 and DE22743.

Address correspondence to: Yong-Jing Gao, Institute of Nautical Medicine, Nantong University, 9 Seyuan Road, Nantong, Jiangsu 226019, China. Phone: 86.513.55003374; E-mail: gaoyongjing@ hotmail.com or gaoyongjing@ntu.edu.cn.
1. Charo IF, Ransohoff RM. The many roles of chemokines and chemokine receptors in inflammation. N Engl J Med. 2006;354(6):610-621.

2. Asensio VC, Campbell IL. Chemokines in the CNS: plurifunctional mediators in diverse states. Trends Neurosci. 1999;22(11):504-512.

3. Savarin-Vuaillat C, Ransohoff RM. Chemokines and chemokine receptors in neurological disease: raise, retain, or reduce? Neurotherapeutics. 2007;4(4):590-601.

4. Kiguchi N, Kobayashi Y, Kishioka S. Chemokines and cytokines in neuroinflammation leading to neuropathic pain. Curr Opin Pharmacol. 2012;12(1):55-61.

5. Old EA, Malcangio M. Chemokine mediated neuron-glia communication and aberrant signalling in neuropathic pain states. Curr Opin Pharmacol. 2012;12(1):67-73.

6. Imai S, et al. Epigenetic transcriptional activation of monocyte chemotactic protein 3 contributes to long-lasting neuropathic pain. Brain. 2013;136(pt 3):828-843.

7. Zhang ZJ, Cao DL, Zhang X, Ji RR, Gao YJ. Chemokine contribution to neuropathic pain: respective induction of CXCL1 and CXCR2 in spinal cord astrocytes and neurons. Pain. 2013;154(10):2185-2197.

8. Bhangoo SK, et al. CXCR4 chemokine receptor signaling mediates pain hypersensitivity in association with antiretroviral toxic neuropathy. Brain
Behav Immun. 2007;21(5):581-591.

9. Verge GM, Milligan ED, Maier SF, Watkins LR, Naeve GS, Foster AC. Fractalkine (CX3CL1) and fractalkine receptor (CX3CR1) distribution in spinal cord and dorsal root ganglia under basal and neuropathic pain conditions. Eur J Neurosci. 2004;20(5):1150-1160.

10. Gao YJ, et al. JNK-induced MCP-1 production in spinal cord astrocytes contributes to central sensitization and neuropathic pain. J Neurosci. 2009;29(13):4096-4108.

11. Zhang J, De Koninck Y. Spatial and temporal relationship between monocyte chemoattractant protein-1 expression and spinal glial activation following peripheral nerve injury. J Neurochem. 2006;97(3):772-783.

12. Zhao P, Waxman SG, Hains BC. Modulation of thalamic nociceptive processing after spinal cord injury through remote activation of thalamic microglia by cysteine cysteine chemokine ligand 21. J Neurosci. 2007;27(33):8893-8902.

13. Staniland AA, et al. Reduced inflammatory and neuropathic pain and decreased spinal microglial response in fractalkine receptor (CX3CR1) knockout mice. J Neurochem. 2010;114(4):1143-1157.

14. Ansel KM, et al. A chemokine-driven positive feedback loop organizes lymphoid follicles. Nature. 2000;406(6793):309-314.

15. Forster R, Mattis AE, Kremmer E, Wolf E, Brem
G, Lipp M. A putative chemokine receptor, BLR1, directs $B$ cell migration to defined lymphoid organs and specific anatomic compartments of the spleen. Cell.1996;87(6):1037-1047.

16. Bagaeva LV, Rao P, Powers JM, Segal BM. CXC chemokine ligand 13 plays a role in experimental autoimmune encephalomyelitis. JImmunol. 2006;176(12):7676-7685.

17. Magliozzi R, Columba-Cabezas S, Serafini B, Aloisi F. Intracerebral expression of CXCL13 and BAFF is accompanied by formation of lymphoid follicle-like structures in the meninges of mice with relapsing experimental autoimmune encephalomyelitis. J Neuroimmunol. 2004;148(1-2):11-23.

18. Smith JR, Braziel RM, Paoletti S, Lipp M, Uguccioni M, Rosenbaum JT. Expression of B-cellattracting chemokine 1 (CXCL13) by malignant lymphocytes and vascular endothelium in primary central nervous system lymphoma. Blood. 2003;101(3):815-821.

19. Kim CH, Rott LS, Clark-Lewis I, Campbell DJ, Wu L, Butcher EC. Subspecialization of CXCR5 $T$ cells: $B$ helper activity is focused in a germinal center-localized subset of CXCR $5^{+} \mathrm{T}$ cells. J Exp Med. 2001;193(12):1373-1381.

20. Krumbholz M, et al. Chemokines in multiple sclerosis: CXCL12 and CXCL13 up-regulation is differentially linked to CNS immune cell recruitment. Brain. 2006;129(Pt 1):200-211.

21. Sellebjerg F, et al. Increased cerebrospinal fluid 
concentrations of the chemokine CXCL13 in active MS. Neurology. 2009;73(23):2003-2010.

22. Ljostad U, Mygland A. CSF B-lymphocyte chemoattractant (CXCL13) in the early diagnosis of acute Lyme neuroborreliosis. J Neurol. 2008;255(5):732-737.

23. Fischer L, et al. CXCL13 and CXCL12 in central nervous system lymphoma patients. Clin Cancer Res. 2009;15(19):5968-5973.

24. Rainey-Barger EK, Rumble JM, Lalor SJ, Esen N, Segal BM, Irani DN. The lymphoid chemokine, CXCL13, is dispensable for the initial recruitment of B cells to the acutely inflamed central nervous system. Brain Behav Immun. 2011;25(5):922-931.

25. Kawasaki Y, et al. Distinct roles of matrix metalloproteases in the early- and late-phase development of neuropathic pain. Nat Med. 2008;14(3):331-336.

26. Bartel DP. MicroRNAs: target recognition and regulatory functions. Cell. 2009;136(2):215-233.

27. Zhou L, Qi X, Potashkin JA, Abdul-Karim FW, Gorodeski GI. MicroRNAs miR-186 and miR-150 down-regulate expression of the pro-apoptotic purinergic $\mathrm{P} 2 \mathrm{X} 7$ receptor by activation of instability sites at the 3 '-untranslated region of the gene that decrease steady-state levels of the transcript. J Biol Chem. 2008;283(42):28274-28286.

28. Ji RR, Kohno T, Moore KA, Woolf CJ. Central sensitization and LTP: do pain and memory share similar mechanisms? Trends Neurosci. 2003;26(12):696-705.

29. Wang Z, et al. Pronociceptive actions of dynorphin maintain chronic neuropathic pain. J Neurosci. 2001;21(5):1779-1786.

30. Tian Y, Wang S, Ma Y, Lim G, Kim H, Mao J. Leptin enhances NMDA-induced spinal excitation in rats: A functional link between adipocytokine and neuropathic pain. Pain. 2011;152(6):1263-1271.

31. Ji XT, et al. Spinal astrocytic activation contributes to mechanical allodynia in a rat chemotherapy-induced neuropathic pain model. PLoS One. 2013;8(4):e60733.

32. Zheng FY, Xiao WH, Bennett GJ. The response of spinal microglia to chemotherapy-evoked painful peripheral neuropathies is distinct from that evoked by traumatic nerve injuries. Neuroscience. 2011;176:447-454.

33. Zhang H, Yoon SY, Dougherty PM. Evidence that spinal astrocytes but not microglia contribute to the pathogenesis of Paclitaxel-induced painful neuropathy. J Pain. 2012;13(3):293-303.

34. Liao YH, et al. Spinal astrocytic activation contributes to mechanical allodynia in a mouse model of type 2 diabetes. Brain Res. 2011;1368:324-335.

35. Zhuang ZY, Gerner P, Woolf CJ, Ji RR. ERK is sequentially activated in neurons, microglia, and astrocytes by spinal nerve ligation and contributes to mechanical allodynia in this neuropathic pain model. Pain. 2005;114(1-2):149-159.

36. Cao DL, Zhang ZJ, Xie RG, Jiang BC, Ji RR, Gao YJ. Chemokine CXCL1 enhances inflammatory pain and increases NMDA receptor activity and COX-2 expression in spinal cord neurons via activation of CXCR2. Exp Neurol. 2014;261:328-336.
37. Lu Y, et al. TRAF6 upregulation in spinal astrocytes maintains neuropathic pain by integrating TNF- $\alpha$ and IL-1 $\beta$ signaling. Pain. 2014;155(12):2618-2629.

38. Gao YJ, Zhang L, Ji RR. Spinal injection of TNF- $\alpha$-activated astrocytes produces persistent pain symptom mechanical allodynia by releasing monocyte chemoattractant protein-1. Glia. 2010;58(15):1871-1880.

39. Ramesh G, et al. Interaction of the Lyme disease spirochete Borrelia burgdorferi with brain parenchyma elicits inflammatory mediators from glial cells as well as glial and neuronal apoptosis. Am J Pathol. 2008;173(5):1415-1427.

40. Ramesh G, et al. Possible role of glial cells in the onset and progression of Lyme neuroborreliosis. JNeuroinflammation. 2009;6:23.

41. Ji RR, Berta T, Nedergaard M. Glia and pain: is chronic pain a gliopathy? Pain. 2013; 154(suppl 1):S10-S28.

42. White FA, Jung H, Miller RJ. Chemokines and the pathophysiology of neuropathic pain. Proc Natl Acad Sci U S A. 2007;104(51):20151-20158.

43. Narayan K, et al. The nervous system as ectopic germinal center: CXCL13 and IgG in lyme neuroborreliosis. Ann Neurol. 2005;57(6):813-823.

44. Sakai A, Saitow F, Miyake N, Miyake K, Shimada T, Suzuki H. miR-7a alleviates the maintenance of neuropathic pain through regulation of neuronal excitability. Brain. 2013;136(pt 9):2738-2750.

45. Park CK, et al. Extracellular microRNAs activate nociceptor neurons to elicit pain via TLR7 and TRPA1. Neuron. 2014;82(1):47-54.

46. Cai J, et al. miR-186 downregulation correlates with poor survival in lung adenocarcinoma, where it interferes with cell-cycle regulation. Cancer Res. 2013;73(2):756-766.

47. Kobayashi K, Takahashi E, Miyagawa Y, Yamanaka H, Noguchi K. Induction of the P2X7 receptor in spinal microglia in a neuropathic pain model. Neurosci Lett. 2011;504(1):57-61.

48. He WJ, et al. Spinal P2X(7) receptor mediates microglia activation-induced neuropathic pain in the sciatic nerve injury rat model. Behav Brain Res. 2012;226(1):163-170.

49. Ries J, et al. miR-186, miR-3651 and miR-494: potential biomarkers for oral squamous cell carcinoma extracted from whole blood. Oncol Rep. 2014;31(3):1429-1436.

50. Ji RR, Malcangio M, Strichartz GR. MAP kinase and pain. Brain Res Rev. 2009;60(1):135-148.

51. Berta T, Liu YC, Xu ZZ, Ji RR. Tissue plasminogen activator contributes to morphine tolerance and induces mechanical allodynia via astrocytic IL-1beta and ERK signaling in the spinal cord of mice. Neuroscience. 2013;247:376-385.

52. Kawasaki Y, Zhang L, Cheng JK, Ji RR. Cytokine mechanisms of central sensitization: distinct and overlapping role of interleukin-1beta, interleukin-6, and tumor necrosis factor-alpha in regulating synaptic and neuronal activity in the superficial spinal cord. J Neurosci. 2008;28(20):5189-5194.

53. Harrison JK, et al. Role for neuronally derived fractalkine in mediating interactions between neurons and CX3CR1-expressing microglia. Proc
Natl Acad Sci U S A. 1998;95(18):10896-10901.

54. Lindia JA, McGowan E, Jochnowitz N, Abbadie C. Induction of CX3CL1 expression in astrocytes and CX3CR1 in microglia in the spinal cord of a rat model of neuropathic pain. J Pain. 2005;6(7):434-438.

55. Raghavendra V, Tanga FY, DeLeo JA. Complete Freunds adjuvant-induced peripheral inflammation evokes glial activation and proinflammatory cytokine expression in the CNS. Eur J Neurosci. 2004;20(2):467-473.

56. Svensson M, Eriksson NP, Aldskogius H. Evidence for activation of astrocytes via reactive microglial cells following hypoglossal nerve transection. J Neurosci Res. 1993;35(4):373-381.

57. Tanga FY, Raghavendra V, DeLeo JA. Quantitative real-time RT-PCR assessment of spinal microglial and astrocytic activation markers in a rat model of neuropathic pain. Neurochem Int. 2004;45(2-3):397-407.

58. Miyoshi K, Obata K, Kondo T, Okamura H, Noguchi K. Interleukin-18-mediated microglia/ astrocyte interaction in the spinal cord enhances neuropathic pain processing after nerve injury. J Neurosci. 2008;28(48):12775-12787.

59. Berta T, et al. Extracellular caspase- 6 drives murine inflammatory pain via microglial TNF- $\alpha$ secretion. J Clin Invest. 2014;124(3):1173-1186.

60. Abbadie C, et al. Impaired neuropathic pain responses in mice lacking the chemokine receptor CCR2. Proc Natl Acad Sci U S A. 2003;100(13):7947-7952.

61. Zhang J, Shi XQ, Echeverry S, Mogil JS, De Koninck Y, Rivest S. Expression of CCR2 in both resident and bone marrow-derived microglia plays a critical role in neuropathic pain. J Neurosci. 2007;27(45):12396-12406.

62. Huang $\mathrm{C}$, et al. Critical role of connexin 43 in secondary expansion of traumatic spinal cord injury. J Neurosci. 2012;32(10):3333-3338.

63. Tsuda M, et al. P2X4 receptors induced in spinal microglia gate tactile allodynia after nerve injury. Nature. 2003;424(6950):778-783.

64. Monif M, Reid CA, Powell KL, Smart ML, Williams DA. The P2X7 receptor drives microglia activation and proliferation: a trophic role for P2X7R pore. J Neurosci. 2009;29(12):3781-3791.

65. Kim SH, Chung JM. An experimental model for peripheral neuropathy produced by segmental spinal nerve ligation in the rat. Pain. 1992;50(3):355-363.

66. Hu HJ, Glauner KS. ERK integrates PKA and PKC signaling in superficial dorsal horn neurons. J Neurophysiol. 2003;90(3):1671-1679.

67. Obernosterer G, Martinez J, Alenius M. Locked nucleic acid-based in situ detection of microRNAs in mouse tissue sections. Nat Protoc. 2007;2(6):1508-1514

68. Coull JA, et al. BDNF from microglia causes the shift in neuronal anion gradient underlying neuropathic pain. Nature. 2005;438(7070):1017-1021.

69. Kobayashi K, Yamanaka H, Fukuoka T, Dai Y, Obata K, Noguchi K. P2Y12 receptor upregulation in activated microglia is a gateway of p38 signaling and neuropathic pain. J Neurosci. 2008;28(11):2892-2902. 